Enforcement by Hearing:

An Integrated Model of Evidence Production

by

Chris William Sanchirico, Columbia University

December 1997

December 1995, Revised Jamarary 1998

Discussion Paper Series No. 9798-05

de 9798.05
pages 66 


\title{
ENFORCEMENT BY HEARING: AN INTEgRated MOdEL OF EVIDENCE Production ${ }^{1}$
}

\author{
Chris William Sanchirico
}

First Draft: December 1995

This Draft: ${ }^{2}$ December 1997

\author{
Columbia University, Department of Economics and School of Law \\ 420 West $118^{\text {th }}$ Street, New York, New York 10027 \\ Voice: (212) 854 4037, Fax: (212) 8548059 \\ Email: cs282@columbia.edu, Home Page: www.columbia.edu/ cs282
}

The paper is a theoretical analysis of incentive setting via civil litigation, with a focus on incentives for care in activities that may be harmful to others (torts). It makes two main contributions: one directly policy-relevant, one conceptual.

In most existing research, litigation is modeled in reduced form, as a sort of costly audit, without explicit specification of the fact-finding process. In such models it is always more costly to implement higher levels of care, implying that the second best level of care is lower than the first. Here we explicitly consider the court's information problem (in a single agent model; Sanchirico (1997) considers multiple parties). We find that implementation costs tend to decline in care level and the second best tends to exceed the first. This result in hand, we suggest that the familiar claim that current tort systems "overdeter" may in fact be no indictment.

A second contribution of the paper is its "integrated" conception of evidence production, endogenous cost evidence. Evidence production in court is modeled as costly signaling, where signal costs are endogenous to unobserved choices made outside the court room. Care is inspired to the extent that it reduces signaling costs and so increases payoffs at the subsequent proceeding. These signaling/evidence costs are in turn costs of incentive setting via evidence production. In contrast to existing models and conventional wisdom, this view has the ironic implication that "perfect" (non-falsifiable) evidence may not be good enough: "less perfect" evidence which is sufficiently - but not infinitely-more costly for disobedient actions is likely to be cheaper all around and thus a more efficient means of setting incentives.

While the paper's focus is on tort law, the model it introduces applies generally to situations in which a principal attempts to influence the hidden behavior of an agent based on information that the agent herself supplies.

JEL: K13, K40, K41, D82, D62, C72

Key Words: Evidence Production, Mechanism Design, Litigation, Torts. 
The salience of litigation and tort reform in several nations, ${ }^{3}$ and the resulting demand for accurate facts and figures, has generated a welcome increase in available data and careful data analysis. Yet it is imperative that the growing prevalence of empirical work in this area, not slow the pace of advance in the economic theory of legal suits. The risk is an unbalanced literature in which correlations are well-established, but poorly interpreted; in which hypotheses are well-tested, but poorly chosen.

On the surface, it would seem as if theorizing about legal process is already in abundant and effective supply. Yet a careful examination of the literature reveals a curious deficiency: the economic theory of litigation has surprisingly little to say about what actually happens in court. ${ }^{4}$ Many studies simply assume that the court is able to deduce whatever factual knowledge is necessary to implement the legal rule in question, be that care exercised, harm caused, or some portion of both. ${ }^{5}$ Models that feature asymmetric information as between the litigating parties ${ }^{6}$ simply endow their agents with heterogeneous beliefs about what will happen at trial without specifying the process that produces trial outcomes and presumably founds these beliefs. Even models that explicitly account for trial error start with an exogenous relationship between the probability of error and either investment in the court system ${ }^{7}$ or the preparation effort of the parties. $^{8}$

To be sure, modeling trial in "reduced form" "has been a fruitful research strategy, enabling the literature to focus attention on important issues such as trial preparation, settlement likelihood, settlement amount, and the filing of suit. The problem with exclusive reliance on a reduced form, however, is that one is never sure whether important considerations have been lost in the reduction. This paper shows that opening the black box of the trial process does indeed 
have important and counterintuitive implications for some of the most fundamental issues in civil litigation design.

We present a new model of civil ${ }^{10}$ litigation that integrates a moral hazard analysis of incentives in the underlying activity with an explicit adverse selection model of evidence production at the subsequent proceeding. To fix ideas, we focus on the problem of setting incentives for care in activities that are potentially hazardous to others (e.g., driving, medical practice, product design, or toxic waste disposal) based on evidence produced by the agent herself at a later hearing. The analysis we provide, however, is relevant not just to other areas of law but to any situation in which a principal attempts to influence the hidden behavior of an agent based in whole or part on information that the agent herself supplies. Examples include reports by corporate managers to their boards on issues affected by managerial performance.

A fundamental question of incentive setting via civil process concerns the level of care that should be induced. One contribution of this paper is to show that accounting for the costs of the civil litigation system - in an explicit model of evidence production--tends to make it optimal to induce more care than both the first best level (the level that, as in Calabresi (1972), minimizes the costs of accidents and the costs of precaution) Oddly enough, accounting for the "price" of implementing care, makes us want to implement more of it.

More than this, our finding is in marked contrast with the second best outcomes generated by the reduced form models that populate the literature. Most such models are actually some variant of a "costly state verification" 11 " or "audit" model. In the simplest version of such a model, the principal chooses an audit probability and a punishment for disobedience; audits are costly, but perfectly reveal any disobedience. With risk neutral agents, the minimal cost of enforcement of any given level of care always entails imposing maximal fines, as determined, for 
example, by solvency constraints. The probability of audit is then set so that the expected punishment from disobedience exceeds the additional costs of the target level of care over no care. Thus, enforcing higher levels of care, which have higher additional costs, means conducting more frequent audits and thus entails higher implementation costs. As is clear from the schematic in Figure 1, the positive slope of the minimal cost of implementation schedule in turn implies that the second best must be no greater than the first best: care levels higher than the first best have higher Calabresian costs and are also more costly to implement.

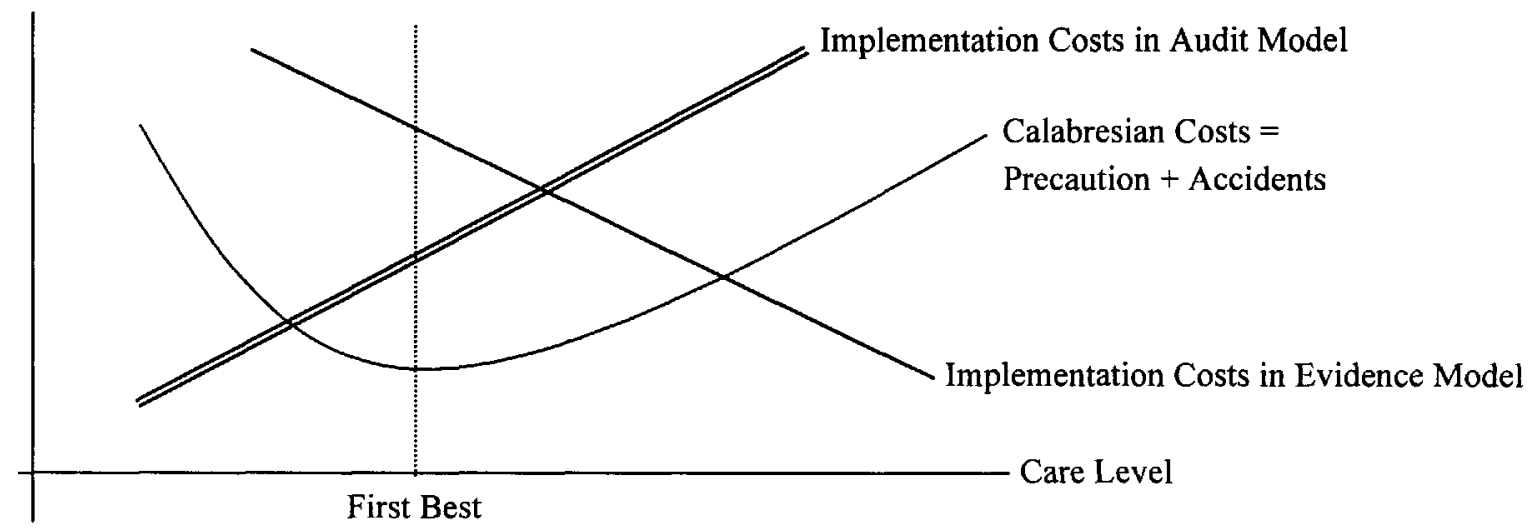

Figure 1: Second Best v. First Best and the Slope of Implementation Costs

In contrast, the minimal cost of implementation in our model tends to decline in the level of care implemented, implying by an argument symmetric to that in the last sentence, that our second best tends to exceed both the first best and the audit model's second best. We say "tends" in the same way we would say that demand "tends" to decrease in price. In particular, we decompose the slope of the minimal cost of implementation schedule into two effects. One, like the substitution effect on demand, is always strictly negative, for all parameters values and 
functional forms (Proposition 3). The other, like the income effect, is patently ambiguous (Example 5; Proposition 4).

This first unambiguously negative effect, which we call the "change in presenter" effect derives from the fact that presentation cost differences are what give evidence meaning in our model-indeed we will argue in any reasonable model. We show that a piece of evidence is useful in setting incentives only to the extent that it is more costly to present if the agent takes less than the desired level of care (Proposition 1). Otherwise, the agent will always do better in both the underlying activity and the hearing by taking less care, no matter how we reward this evidence at the hearing. This means that when we induce agents to take higher levels of care we essentially turn them into lower cost evidence producers. Since the cost of evidence production is a deadweight loss to the system, we implement the higher level of care more cheaply. Of course, the evidence presented may itself change when we implement more care; this leads to the second ambiguous effect, which we term the "change in case."

This purely theoretical point has important practical implications for the debate over tort reform now ongoing in Canada and the United States. ${ }^{12}$ One of the most rhetorically powerful claims marshaled against current systems is that they "overdeter," causing agents to take too much care. In a recent QJE article, for example, Kessler and McClellan (1996) lend careful empirical support to the claim that the threat of suit causes doctors to practice "defensive medicine. ${ }^{13 "}$ Though it is often left ambiguous, those who point to overdeterrence seem to use the first best as their bench mark. ${ }^{14}$ At worst, such commentators have ignored variation in implementation costs and the resulting distinction between first and second best. At best, they have in mind the reduced form audit model of litigation described above, in which the first best care level exceeds the second best, so that care in excess of the first best is excessive in both 
senses. In the more explicit model of fact finding that we propose, however, the second best tends to exceed the first and so some amount of "overdeterrence" relative to the first best is likely to be efficient. At the very least, our model indicates that claims of overdeterrence should be viewed with serious skepticism. To the extent that evidence production is an important element in the current system of litigation, "too much" care may be just the right amount. ${ }^{15}$

Despite its connection to the current policy debate, the paper's chief contribution may be purely conceptual. The paper proposes a new perspective on evidence production- to be sure, a new combination of existing ideas from mechanism design —-that has advantages, in terms of both coherence and realism, over how evidence production has been treated in the literature. That literature sorts roughly into three approaches. The first and oldest approach to evidence production, as typified by the Green Symposium (1986), is the Scientific. This supposes that the court considers evidence the way a scientist considers data, making deductions based on prior beliefs about the correlation of her observations with various underlying truths. The most obvious problem with this approach is that the molecules under the scientist's microscope have no particular interest in what the scientist concludes, nor any ability to alter or influence the conclusion. Neither can be said of the parties to a suit standing before the judge. Naturally, the judge's ruling likely has important implications for the parties' material well-being. Moreover, it inheres in the nature of civil process that in making this ruling the judge relies almost entirely on what these agents choose to place or perform before its bench.

A less obvious problem with the scientific approach is that it neglects the "underlying activity," (the tort or breach that gave rise to the litigation in the first place). The purpose of 
litigation is presumably not to out the truth for truth's sake - as if litigation were an academic endeavor-but to affect how agents behave outside the courtroom in real economic interaction.

The Classic Moral Hazard approach to evidence production, as typified by Hermalin and Katz (1991), solves the second problem of the Scientific approach—regarding incentives in the underlying activity—but not the first-regarding incentives in the courtroom. The analogy here is to the employer, rather than the scientist, who induces her employee to work hard by conditioning wages on an exogenous, noisy signal of work effort, usually understood to be firm output. This approach views evidence as a noisy signal of behavior in the underlying activity. The problem with this analogy is that the employer's signal is exogenously generated (conditional on the employee's choice of effort), whereas the signal received by the court is endogenous in the sense that it is chosen by the parties in interest. Thus, the Classic Moral Hazard approach fails to take into account the second layer of the principal's incentive problem: the incentive of the agent after she has chosen her action, to manipulate the signals upon which the court conditions rewards and punishments.

The third approach to evidence production consists of the No-lying models of Sobel (1985), Milgrom and Roberts (1986), Okuno-Fujiwara, Postlewaite and Suzumura (1990), Froeb and Kobayashi $(1993,1995)$, Lipman and Seppi (1995). These models do account for incentives at the hearing stage, but in a limited way. These models adopt some form of the assumption that agents are unable to lie. Instead, the focus is on agents' incentives to fail to report all that they know. By a now familiar "unraveling" argument, the principal is able to obtain her favorite outcome by assuming the worst for the agent on whatever points the agent leaves ambiguous. Unfortunately, the unraveling argument itself unravels if agents can lie. 
Suppose, for example, that the truth, known only to the agent, is a number between 1 and 10 . The agent wants the principal/decision maker to think the number is as large as possible because this leads her to make the most favorable decision for the agent. The agent can omit to tell the principal all he knows, by reporting a non-singleton subset of values; but he can not lie, so that the interval must contain the true value. Under these assumptions, the principal can always learn the truth by announcing that she will act as if the truth is the lowest number in the subset reported by the agent. The agent will never include numbers lower than the truth in his report. And since he must include the truth itself, the lowest value in his report will be in fact be true. Of course, if the agent can lie the result evaporates, for then he will always report the singleton $\{10\}$ under this rule.

In contrast to the existing literature on evidence production, the object of this paper is to analyze the full double incentive problem, integrating incentives in both the underlying activity and the hearing - and this in a model in which agents can and will lie when it is in their interest to do so. Attaining this ambitious goal means giving up a bit on realism in other areas. Thus we present a single agent model of evidence production in which hearings are periodic and mandatory. There is, for instance, no mention of filing of suit, settlement negotiations, or pretrial discovery. (Sanchirico (1997) extends the model we present here to allow for multiple parties with correlated private information and endogenously derives the private instigation of suits.)

Our analysis differs from the Scientific and Classical Moral Hazard approaches in that we argue for shifting the focus away from differences in pure correlation (with underlying truths or underlying actions) onto differences in presentation costs. In our model, and, we would argue, in actual litigation, all the court can conclude from the agent's presentation of a given piece of 
evidence is that it was in the agent's interest to do so. Evidence is then only useful in setting incentives in the underlying activity to the extent that the agent's interest in presenting it changes with the action she takes in the underlying activity. Since actions in the underlying activity are unobservable by the court, this change cannot come from differences in the liability incurred per each given case. Hence, the change must come from differences in cost (or difficulty) of presentation caused by different actions taken outside the courtroom (Proposition 1). Essentially, then, we suggest viewing the subsequent hearing as a Spence (1974)-style signaling ${ }^{16}$ game in which the agent's "type" (here evidence costs) is potentially affected by her choice of action in the underlying activity. Thus we call this conception "endogenous cost evidence. ${ }^{17}$ ",

This focus on cost rather than correlation distinguishes our approach from the Scientific and Classical Moral Hazard approaches. Our approach is distinguished from the No-lying models most obviously by the fact that we allow agents to lie: agents choose what to perform in court and the choices available include announcements that are simply lies. But even more, it is possible to distinguish our approach from the most favorable, ex post revision of the No-lying approach. To make it (only somewhat) more plausible, one might recast the No-lying assumption as the assumption that "perfect evidence" exists, where evidence is "perfect" for a given target action if it is of infinitely (or near infinitely) higher cost following alternative disobedient actions. Inability (to lie) is thus translated into "infinite cost" and an infinite cost of lying is translated into an infinite cost of presenting a particular perfect case following disobedience. ${ }^{18}$

In the first place, we remark that the very existence of perfect evidence is open to serious question. Here it is crucial to distinguish what happens before the judge from what happens outside the courtroom in the underlying activity. One is tempted to point to the plaintiff's 
missing a leg as an example of essentially perfect evidence. After all, the judge can see the injury with her own eyes. But the missing leg is perfect evidence only of the bare fact that the plaintiff now appears before the court less one leg. It says nothing about how the leg was removed - e.g., whether the injury was caused by the defendant's lack of care. Information about how things happen outside the courtroom is what matters for influencing how people act outside the court room.

In the second place, even if one is willing to accept that perfect evidence occasionally exists, it is quite another thing to claim that it is an important factor in actual litigation. In reality, most evidence consists of cross-examined oral testimony, documents, media and the occasional physical article-all of which are forgeable, at a price.

Lastly, even if and when such perfect evidence is occasionally available, it is important to note that the mere fact that it is "perfect" does not imply that we would want to use it to set incentives. Indeed, "less perfect" evidence which is only sufficiently more costly for disobedient actions is likely to be cheaper all around and thus a more efficient means of setting incentives in the underlying activity (Proposition 2). Thus when one is called on to prove identity in obtaining a new driver's license from the Department of Motor Vehicles, a passport and expired license will suffice. "More perfect" evidence is certainly available—we could require people to present hundreds of forms of identification, procure several witnesses, provide DNA test results, etc... Such evidence, as a package, would be "more perfect" than what is actually required in the sense that the cost of forgery would be much higher. The point, though, is that truthful presentation would also be extremely costly: it might take several days just to gather enough evidence to satisfy the requirements. We would do better, as even the DMV has concluded, by drawing in any slack in incentive constraints and requiring much less to "prove" identity. 
The remainder of the paper substantiates the claims made in the preceding paragraphs.

Section 1 presents the formal model. Section 2 illustrates the basic workings of the model with a simple example. The reader should be able to glean most of the paper's major points from this example. General results are contained in Section 3. Sections 4 and 5 solve the mechanism design problem analytically for two special cases: deterministic multiplicatively separable evidence costs and stochastic multiplicatively separable evidence costs. All proofs appear in an appendix.

\section{The Model}

We introduce the concept of "endogenous cost evidence" in a model of single, agent mandatory hearings. Essentially, a Spence-style signaling game is appended to the usual moral hazard model, with the key ingredient being that "types" in the subsequent signaling game are (stochastically) dependent on action choice in the moral hazard stage.

\subsection{The Model's Three Phases}

A risk neutral agent and a risk neutral principal interact in a model with three phases as laid out in Figure 2. We may think of the principal as the court and the agent as an individual engaged in an activity, such as driving, that is potentially hazardous to others. 


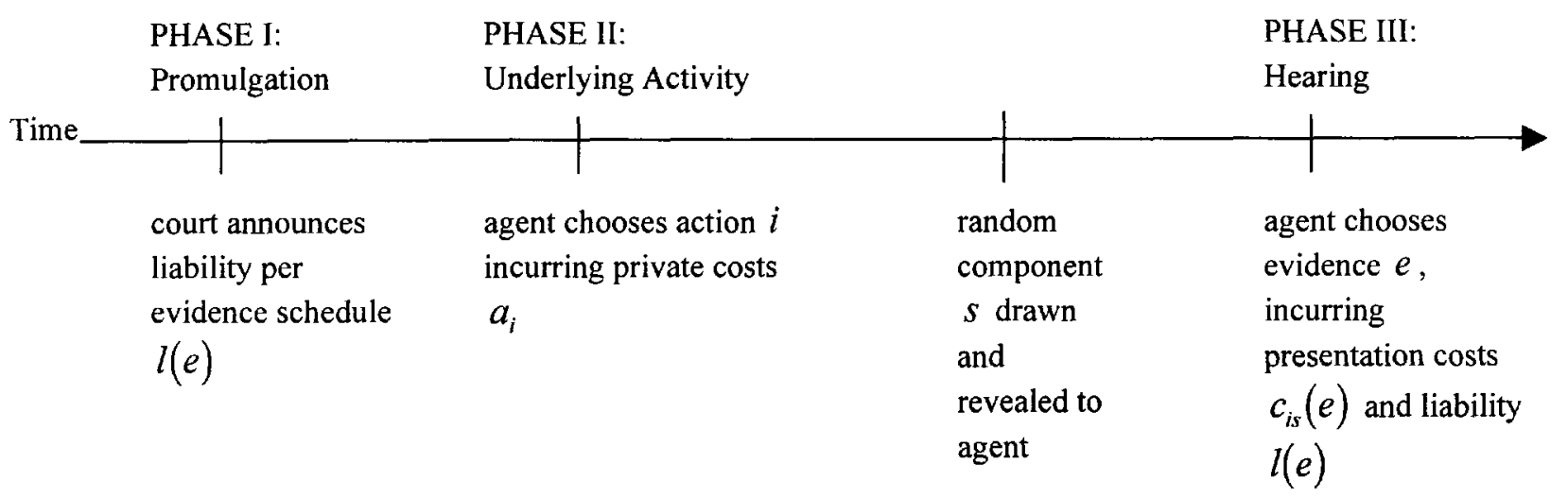

Figure 2: The Model's Three Phases

In the first promulgation phase the principal announces a liability (per evidence) schedule $l: E \rightarrow \Re$. The schedule tells the agent how much she must pay $l(e)$ based on the case or evidence $e \in E$ she presents at the hearing, the third phase, described below. We impose no restrictions, neither ordering nor topological, on $E$.

In the underlying activity, the agent chooses an action $i$ from the $\operatorname{set}^{19} I=\{1, \ldots, I\}$. The agent's private cost for action $i$ is $a_{i}$, where $I$ is arranged so that $a_{1}<a_{2}<\ldots<a_{l}$. The social cost (excluding the agent's private costs) is $h_{i}$.

Following on our automotive interpretation, we may think of $i$ as the level of care that the agent exercises while driving. The variable $a_{i}$ is then her cost of care (netted against any expected accident costs that she herself bears) and $h_{i}$ is the (expected) cost of accidents for others.

Both schedules, $\left(a_{1}, \ldots, a_{I}\right)$ and $\left(h_{1}, \ldots, h_{I}\right)$ are common knowledge throughout, but only the agent observes his actual choice of action. All during the underlying activity the court sits 
waiting behind its bench in a courtroom far from the action; it never observes the agent's choice of $i$, nor does it receive any exogenous signal of $i$.

Some time after her choice of action, the agent appears before the court at the hearing. There the agent chooses what case $e$ to present from the set $E$, balancing the resulting liability under $l(e)$ against the costs, if any, of presentation. The set $E$ contains all possible "performances" that the agent could stage before the court. This includes, for example, the case that consists solely of the cheap talk statement, "I was careful." Importantly, we also allow the possibility that some cases are costly and that-more to the point-the level of these costs is (probabilistically) affected by the agent's choice of action in the underlying activity. The evidence cost schedule for the agent is thus $c_{i s}: E \rightarrow \Re_{+}$with values $c_{i s}(e)$, where $i$ is the agent's chosen action and $s$ is a state or random component. The state $s$ is drawn from the finite set $S=\{1, \ldots, S\}$ according to probabilities ${ }^{20}\left(p_{1}, \ldots, p_{S}\right)>0, \sum_{s} p_{s}=1$. We refer to pairs $(i, s)$ as circumstances. We emphasize that the dependence of evidence costs on actions is not imposed, only allowed: the model encompasses the case where for every case $e, c_{i s}(e)$ is constant across $i$.

The evidence cost schedules $c_{i s}(e)$ and the probabilities $\left(p_{1}, \ldots, p_{S}\right)$ are common knowledge throughout the three phases. The agent learns the actual value of $s$ after choosing her action $i$, but before choosing her evidence $e$. The court never observes the actual value of $s$, just as it never observes the actual value of $i$.

Following on our automotive interpretation, we imagine that the agent chooses how carefully to drive with an understanding of how this tends to affect what actually comes to pass along her route-for example, whether an accident occurs, and if so, what part of it is observed 
by bystanders, what pieces are left on the ground, etc... Uncertainty in the causal relationship between care and incident is modeled by including the random component $s$. This component represents all relevant aspects of the environment not affected by the agent's choice of care-for example, whether a child chases a ball out into the street or whether someone happens to be watching the street from a nearby window. The agent's choice of $i$ together with nature's choice of $s$ determines what actually happens on the street. We then leave open the possibility that what actually happens on the street affects in turn the costs of staging various performances before the court, taking into account both casting (witnesses) and props (real and documentary evidence).

\subsection{The Principal's Mechanism Design Problem}

Left to her own devices, the agent will always choose the action $i=1$ with lowest private costs; social costs are of no concern to her. The court's problem, in general terms, is to somehow induce the agent to take social costs into account solely through the manner in which it rewards the evidence that the agent chooses to produce.

To understand the court's problem in more detail, suppose it is considering promulgating a particular liability schedule $l(e)$. Though it never sees anything more than the evidence actually presented by the agent at the hearing, the court can figure out how the agent would react to this schedule in every circumstance $(i, s)$. In particular, since it knows the agent's evidence cost schedule $c_{i s}$ in each circumstance, it can predict how the agent will solve her problem of choosing evidence to minimize the sum of liability and evidence costs, $l(e)+c_{i s}(e)$. Thus, it can calculate the value of the solution $\min _{e}\left[l(e)+c_{i s}(e)\right]$ for each $(i, s)$. Taking the expectation of these values across $s$ for each action $i$, it can then compare the schedule of expected hearing 
payoffs with the schedule of private costs $a_{i}$ in order to determine which action the agent will choose. (For future reference, this is (2) below.)

Once it knows which action the agent will choose, it can go back and calculate the cost of liability schedule $l(e)$ in terms of the expected cost to the agent of the evidence she ends up presenting (for future reference, this corresponds to (3)). Combining these expected evidence costs with the private and social costs of the action actually taken, it can calculate the total social cost resulting from imposition of this particular liability schedule (this is (1)). Note that liability payments are transfers and so do not enter social welfare.

This is how the court analyzes each individual liability schedule. The court's problem is to do such a calculation for all schedules and then choose the one with the lowest sum of private costs, accident costs, and expected evidence costs.

In translating the preceding discussion into formal terms, we need to account for the fact that any given $l(e)$ may make the agent indifferent in choosing her evidence and/or action.

Following convention, we proceed as if the principal can choose how to resolve such ties. Thus, the principal's natural problem is to

Choose $l(e), i$ and $\left(e_{1}, \ldots, e_{s}\right)$ to

$$
\begin{array}{r}
\text { minimize } a_{i}+h_{i}+\sum_{s=1}^{S} p_{s} c_{i s}\left(e_{s}\right) \text { subject to } \\
i \text { minimizes } a_{i}+\sum_{s=1}^{S} p_{s}\left[\min _{e}\left(l(e)+c_{i s}(e)\right)\right], \\
\forall s=1, \ldots, S, \quad e_{s} \text { minimizes } l(e)+c_{i s}(e)
\end{array}
$$

It is more convenient to work an alternative formulation whose equivalence to (1)-(3) is established in Proposition 10. In this alternative formulation, we proceed as if the principal 
chooses both the agent's action and her evidence and liability in each circumstance, subject to two respective incentive constraints, one for the underlying activity and one for the hearing. We represent the choice of liability and evidence in each circumstance by a vector $(l, e)=\left(l_{11}, \ldots, l_{i s}, \ldots, l_{I S} ; e_{11}, \ldots, e_{i s}, \ldots, e_{I S}\right) \in \mathfrak{R}^{I S} \times E^{I S}$, which we also refer to as the liability per evidence schedule. Thus, the principal's direct bifurcated problem is to

$$
\begin{aligned}
& \text { Choose } i \text { and }(l, e)=\left(l_{11}, \ldots, l_{I S} ; e_{1}, \ldots, e_{I S}\right) \text { to } \\
& \text { minimize } a_{i}+h_{i}+\sum_{s=1}^{S} p_{s} c_{i s}\left(e_{i s}\right) \text {, subject to } \\
& \forall i^{\prime}=1, \ldots, I, \quad a_{i}+\sum_{s=1}^{S} p_{s}\left(l_{i s}+c_{i s}\left(e_{i s}\right)\right) \leq a_{i^{\prime}}+\sum_{s=1}^{S} p_{s}\left(l_{i^{\prime} s}+c_{i^{\prime}}\left(e_{i^{\prime}}\right)\right) \\
& \forall\left(i^{\prime}, s^{\prime}\right),\left(i^{\prime \prime}, s^{\prime \prime}\right), \quad \quad l_{i^{\prime} s^{\prime}}+c_{i^{\prime} s^{\prime}}\left(e_{i^{\prime} s^{\prime}}\right) \leq l_{i^{\prime \prime} s^{\prime \prime}}+c_{i^{\prime} s^{\prime}}\left(e_{i^{\prime \prime} s^{\prime \prime}}\right)
\end{aligned}
$$

Constraints (5) and (6) constitute a bifurcation of the incentive compatibility constraint. Constraint (6) says that if the agent were to take action $i^{\prime}$ and observe realization $s^{\prime}$, she would (weakly) prefer the liability and evidence pair $\left(l_{i^{\prime} s^{\prime}}, e_{i^{\prime} s^{\prime}}\right)$ to that assigned to every alternative circumstance $\left(i^{\prime \prime}, s^{\prime \prime}\right)$. We call (6) the hearing constraint. Constraint (5) says that the agent must (weakly) prefer to take action $i$ over all alternative actions $i^{\prime}$, given that she would do what is intended for her in each circumstance at the hearing. We call (5) the underlying activity constraint.

The direct bifurcated formulation is just another way of writing the natural problem. Roughly, if $l(e)$ satisfies the natural problem's constraints (with action $i)$, then the vector $(l, e)$ describing the evidence that would be presented and the liability that would be incurred by the agent in each circumstance will satisfy the direct problem's constraints and return the same 
objective function value. Conversely, if $i$ and $(l, e)$ satisfy the direct problem's constraints, then it is possible to construct a function $l(e)$ consistent with the correspondence between evidence and liability implicit in $(l, e)$ that will satisfy the natural problem's constraints and return the same objective function value. This is formalized in Proposition 10 located in the appendix.

When $i$ and $(l, e)$ satisfy both constraints (5) and (6), we say that $(l, e)$ implements $i$. Action $i$ is said to be implementable if we can find some $(l, e)$ implementing $i$. We also identify the court's sub-problem of implementing a fixed action $i$ at least cost:

Choose $(l, e)$ to

$$
\text { minimize } \sum_{s=1}^{S} p_{s} c_{i s}\left(e_{i s}\right) \text { subject to (5) and (6). }
$$

Given $i$, we denote the solution value of this problem as $C_{i}$ (where $C_{i}=\infty$ if $i$ is not implementable). Clearly action $i$ solves the overall problem (4)-(6) with some $(l, e)$ if and only if it minimizes $a_{i}+h_{i}+C_{i}$.

Remark 1 [Budget Balance]: The reader will notice that we impose no budget balance constraint on liability payments (which may be negative). However, adding the same constant to each $l_{i^{\prime} s^{\prime}}$ has no effect on either the constraints or the objective. Thus the principal can always obtain expected budget balance, given the implemented action $i$, by translating $\left(l_{11}, \ldots, l_{I S}\right)$ so that $\sum_{s=1}^{S} p_{s} l_{i s}=0$. Given a large population of agents, the principal can achieve almost sure budget balance if $s$ is drawn in a manner that allows application of a strong law of large numbers (e.g., independently). 


\subsubsection{Three Issues that will be Carried Throughout}

The principal's problem poses three issues:

1) Implementability: When is it possible to induce the agent to take a given action in the underlying activity solely through observing the evidence she presents at the hearing? Mathematically, when does action $i$ satisfy the incentive constraints (5) and (6) with some liability schedule $(l, e)$ ?

2) Minimal Cost Implementation: Given that an action can be implemented by some liability schedule, what is the cheapest way to do so in terms of evidence costs actually incurred? This is the principal's sub-problem (7).

3) Which Action to Implement: Given the cheapest way to implement each implementable action, which action should society choose?

\section{EXAMPLE}

The agent chooses from ten possible levels of care $i=1, \ldots, 10$ where her private costs are $a_{i}=i$. At the hearing, the agent chooses evidence from $E=\Re_{+}$, where evidence costs are $c_{i}(e)=(11-i) e$. Thus the least amount of care $i=1$ results with certainty in highest evidence costs $10 e$, and vice versa. (Since evidence costs are a deterministic function of actions in this example-i.e., $S$ is a singleton-we drop all notation pertaining to the state).

With these assumptions, the underlying activity and hearing constraints (5) and (6) reduce to

$\forall i^{\prime}=1, \ldots, 10$

$$
\begin{gathered}
i+\left(l_{i}+(11-i) e_{i}\right) \leq i^{\prime}+\left(l_{i^{\prime}}+\left(11-i^{\prime}\right) e_{i^{\prime}}\right) \\
l_{i^{\prime}}+\left(11-i^{\prime}\right) e_{i^{\prime}} \leq l_{i^{\prime \prime}}+\left(11-i^{\prime}\right) e_{i^{\prime \prime}}
\end{gathered}
$$

Rearranging (8) and defining and substituting for hearing payoffs $v_{i^{\prime}}=-l_{i^{\prime}}-\left(11-i^{\prime}\right) e_{i^{\prime}}$ yields: $\forall i^{\prime}=1, \ldots, 10$

$$
a_{i}-a_{i^{\prime}}=i-i^{\prime} \leq v_{i}-v_{i^{\prime}}
$$


Thus to induce the agent to take care level $i$ over some lower level, we must offer her sufficiently higher hearing payoffs in return for her additional caution. Conversely, to insure that she is not over cautious, the return in terms of additional hearing payoffs to increasing care above $i$ must not exceed the additional private cost. (Note as an aside that this two-sided restriction does not appear in the many models of litigation that posit only two levels of care, "careful" and "careless.")

\subsection{Implementability}

Fix a care level $i \geq 2$. Can we find any liability per evidence schedule that is incentive compatible at the hearing (satisfies (9)) and results in hearing payoffs $v_{1}, \ldots, v_{l}$ satisfying (10) viz. this action $i$ ? The answer is yes. Consider assigning all actions $i^{\prime}=1, \ldots, 10$ the same evidence of $e=1$ and the same liability ${ }^{21}$ of $l=0$. Since the agent has no alternative choices for evidence, this schedule is trivially incentive compatible at the hearing. ${ }^{22}$ Moreover, each $i^{\prime}$ obtains payoffs $v_{i^{\prime}}=-l_{i^{\prime}}-\left(11-i^{\prime}\right) e_{i^{\prime}}=-\left(11-i^{\prime}\right)$ at the hearing and this satisfies (10): increasing (decreasing) care by $n$ "units" results in $n$ more (less) dollars of private costs (since $a_{i^{\prime}}=i^{\prime}$ ) and this is exactly compensated for with $n$ more (less) dollars of hearing payoffs $v_{i^{\prime}}=-\left(11-i^{\prime}\right)$.

Note that this liability schedule does not "separate" by choice of evidence; the agent chooses $e=1$ in all circumstances. It does, however, "separate" by hearing payoffs $v_{i^{\prime}}$, and in implementing actions in the underlying activity, this is our only concern.

The reader should not conclude from this example that implementability is universal for all parameter values. A slight reduction in the private cost of the highest action from 10 to 9.99 will mean that no middling action $2 \leq i \leq 9$ is implementable. To see this particular point it is best to consider hearing and underlying activity constraints in combination. Implementability 
requires that the agent have no incentive to take the next lowest level of care $i-1$ in the underlying activity, and then go to the hearing pretending to have taken $i$. For general liability schedules, $(l, e)$ this particular mix of "disobedience and dishonesty" yields payoffs of $-(i-1)+\left(-l_{i}-(11-(i-1)) e_{i}\right)$. We require that this must not exceed payoffs from obediently choosing care level $i$ and then truthfully acting like $i$ at the hearing: $-i+\left(-l_{i}-(11-i) e_{i}\right)$. This requirement reduces to the inequality $e_{i} \geq 1$. However, given our reduction of $a_{10}, e_{i} \geq 1$ implies that the agent will have an incentive to take action $i=10$ and then pretend to have taken action $i$ at the hearing. For this plan yields payoffs of $-9.99+\left(-l_{i}-(11-10) e_{i}\right)=-9.99-l_{i}-e_{i}$, while playing it straight yields $-i+\left(-l_{i}-(11-i) e_{i}\right)$. The net advantage from deviating is then $(10-i) e_{i}-(9.99-i)$, which is strictly positive when $e_{i} \geq 1$. In sum, the evidence needed to induce action $i$ over $i-1$ will also induce $i=10$ over $i$. (Again note that this sort of problem can only arise in models in which action choice is not binary.)

\subsection{Minimal Cost Implementation of a Given Action}

Raise $a_{10}$ back to its original value (i.e., 10 ) and consider the question: among the liability schedules that implement any given $i \geq 2$, which do so at least cost? Actually, the simple schedule used above to show $i$ 's implementability is one answer. The cost of implementing $i$ under that schedule is $c_{i}\left(e_{i}\right)=(11-i) e_{i}$ and we saw in the last paragraph that implementing $i$ over $i-1$ requires $e_{i} \geq 1$. Thus, no implementing schedule can be cheaper than our simple schedule, ${ }^{23}$ in which $e_{i}=1$. 
Now, all that has been said in the last several paragraphs applies to all care levels except the lowest, for which implementation at zero cost is trivial. We thus have a schedule describing the minimum cost of implementing each level of care, as depicted by the solid line in Figure 3:

$$
C_{i}=\left\{\begin{array}{cc}
0 & i=1 \\
11-i & i \geq 2
\end{array}\right. \text {. }
$$

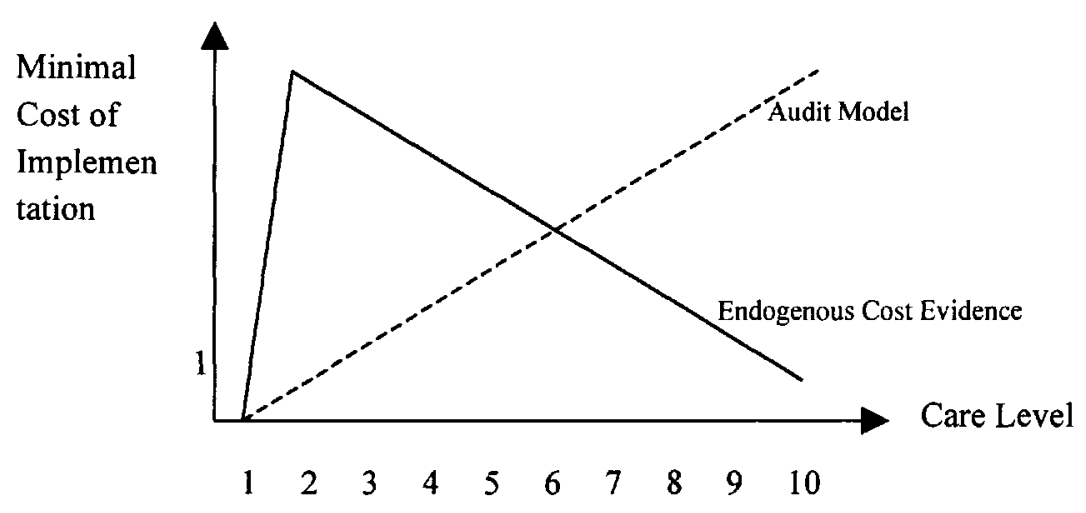

Figure 3: The Minimal Cost of Implementation Schedule

To contrast this result with what is implied by existing models of litigation, consider a random audit model with maximum (net) fine $\bar{F} \in \mathfrak{R}_{++}$and adjustable audit probability ${ }^{24}$ $q \in[0,1]$. Suppose, without saying how, that an audit reveals to the court the injurer's level of care. For simplicity assume that the number $\bar{F}$ is also the social cost of an audit so that expected auditing costs, given $q$, are $q \vec{F}$. In any optimal solution, the court will fine the injurer the maximal amount for not exercising the proper amount of care and set the audit probability as low as possible while still satisfying (10) (with $v_{i^{\prime}}=\bar{F}$ if $i^{\prime} \neq i$ and 0 otherwise). This implies

$$
\left[\forall i^{\prime}=1, \ldots, 10, q \bar{F}=v_{i}-v_{i^{\prime}} \geq a_{i}-a_{i^{\prime}}\right] \Rightarrow q=\frac{i-1}{\bar{F}} .
$$


Thus the minimal cost of implementing any given $i$ (including $i=1$ ) is $q \bar{F}=\frac{i-1}{\bar{F}} \bar{F}=i-1$ as depicted by the upward sloping dotted line in Figure 3.

\subsection{Which Action to Implement}

Given the minimum cost of implementing each level of care, which care level should the court implement? Answering this requires specifying accident costs. Let us suppose that expected harm is given by $h_{i}=\frac{25}{i}$ so that the marginal social benefit of care is $-\left(h_{i}-h_{i-1}\right)=\frac{25}{i(i-1)}$, positive decreasing. Not accounting for the cost of implementation, $C_{i}$, the court would choose care to minimize $i+\frac{25}{i}$, yielding a first best level of $i=5$. This is the level of care prescribed by the classic analysis of tort law (e.g. Calabresi (1972) and Shavell (1980)).

Under the random audit model, the court chooses $i$ to minimize $\left(i+\frac{25}{i}\right)+(i-1)=2 i+\frac{25}{i}$, yielding a second best optimum of $i=4$. Thus, in accord with intuition, the second best is less than the first. Indeed, whatever parameter values we present, the second best will be no more than the first in the audit framework since the cost of implementation is upward sloping.

The story is quite different in our model. Here the court chooses $i$ to minimize $a_{i}+h_{i}+C_{i}=i+\frac{25}{i}+C_{i}$. In the range $i \geq 2$, substitution from (11) reduces the court's objective to $i+\frac{25}{i}+11-i=\frac{25}{i}+11$. The optimum among $i \geq 2$ is then the maximum level of care $i=10$. Since $i=10$ also yields higher payoffs than $i=1\left(-10-\frac{25}{10}-(11-10)=-13.5\right.$ versus $-1-25-0=-26)$ maximal care is indeed socially optimal. Comparing with the middling solution $i=5$ when $C_{i}$ is ignored, we obtain the somewhat paradoxical result that accounting for the cost of implementing care induces the court to implement more of it. Note also that our explicitization of how the court obtains its information produced for us a very different result than the typical audit framework in which second best care is less than the first best. ${ }^{25}$ 


\section{General Results: Comparison with Alternative Models}

In this section we generalize the main points made in the previous example. Our objective here is to characterize the model's structure, independent of any functional form assumptions on evidence costs, in a manner that makes clear the conceptual distinction between the model of evidence and litigation proposed in this paper and the several that exist in the literature.

\subsection{Implementability: Cost versus Correlation}

When the signals received by the court are exogenously generated, as in the Scientific and Classical Moral Hazard approaches, ${ }^{26}$ all that matters is the manner in which signal outcomes are correlated with the underlying truth (including the action actually taken). When as in our model, the agent has full control over the signals received by the court, correlation makes way for cost differences as the chief consideration. In assessing this focus on presentation costs, the reader may ask: does such evidence really exist; and even if it does, do real courts actually make use of it in affecting behavior? We think the answer is yes on both scores. But rather than arguing inductively with a battery of examples, we argue generally, by contradiction. Our claim is that if this sort of evidence does not exist or is not used, the principal has no hope of influencing the agent's incentives in the underlying activity. This is the content of Proposition 1 and its corollaries. 
Proposition 1 [Cost Differences are Necessary for Implementability]: Action $i \geq 2$ is implementable, ONLY IF for some evidence plan $\left(e_{1}, \ldots, e_{s}\right)$ the expected costs savings from choosing $i$ over any alternative action $i^{\prime}$ exceed the additional private costs.

Formally, $i$ is implementable, only if $\exists\left(e_{1}, \ldots, e_{S}\right) \in E^{S}$ s.t.

$$
\forall i^{\prime}=1, \ldots, I \quad \sum_{s=1}^{s} p_{s}\left(c_{i^{\prime}}\left(e_{s}\right)-c_{i s}\left(e_{s}\right)\right) \geq a_{i}-a_{i^{\prime}}
$$

In particular, if $(l, e)$ implements $i,\left(e_{i 1}, \ldots, e_{i S}\right)$ will satisfy (12).

The basic idea behind the Proposition 1 has two steps. When rational parties choose what evidence to present in court they balance two considerations: the cost of presentation (if any) and the extent to which doing so will change the court's ruling in their favor. Hypothetically, two agents (or the same agent in two different circumstances) with the same evidence costs who face the same liability schedule will end up with the same payoffs from the hearing. Their optimization problems are the same. So then are their best possible payoffs.

Stepping back to the underlying activity, in choosing whether to exercise care the agent balances her private costs against her forecast of how taking care will improve the best she can do at the hearing. The court only induces caution when the agent believes that exercising care will increase how well she can do at the hearing by more than it increases her private costs. But by our reasoning in the previous paragraph, taking care can only improve the agent's hearing payoffs if it changes the optimization problem that she faces at the hearing to her benefit. Since the liability schedule $l(e)$ is fixed viz. care choice, any favorable change must come via a favorable shift in evidence costs. Conversely, if her evidence costs are fixed with respect to care choice (e.g., are always zero or negligible) then so are her best hearing payoffs. All "evidence" 
is therefore "cheap talk" and has no effect on care incentives. In this case, private costs are the only consideration in choosing whether to exercise care, and thus the agent is always careless.

Corollary 1: No action save the lowest $i=1$ is implementable if evidence costs do not vary in action, i.e. if $c_{i s}=c_{i^{\prime} s}$ for all $i, i^{\prime}=1, \ldots, I$ and all $s=1, \ldots, S$.

Corollary 2: In a deterministic model (one with a single state) the condition in Proposition 1, which reduces to $\exists e \in E$ s.t. $\forall i^{\prime}, c_{i^{\prime}}(e)-c_{i}(e) \geq a_{i}-a_{i^{\prime}}$, is NECESSARY AND SUFFICIENT for implementability of action $i$.

Example 1 [Application to Section 2's Example]: The example in Section 2 illustrated how cost differences could be used to affect incentives for care. The point of this subsection is to show that this is the only way. That deterministic example satisfies the necessary and sufficient condition in Corollary 2 for every $i=1, \ldots, 10$. In particular, the solution $e_{i}=1$ satisfies (6), which there reduces to $\forall i^{\prime}, c_{i^{\prime}}\left(e_{i}\right)-c_{i}\left(e_{i}\right) \geq a_{i}-a_{i^{\prime}} \Leftrightarrow\left(i-i^{\prime}\right) e_{i} \geq i-i^{\prime}$. Conversely, return to the alteration in Section 2.1 wherein we reduced $a_{10}$ to 9.99 and showed that no middling $2 \leq i \leq 9$ is implementable. Accordingly, when $2 \leq i \leq 9$, no evidence $e$ will satisfy (6). When the alternative action is $i^{\prime}=i-1 \geq 1,\left(i-i^{\prime}\right) e \geq a_{i}-a_{i^{\prime}}$ implies $e \geq 1$. When the alternative action is $i^{\prime}=10$ we have $(i-10) e \geq i-9.99 \Leftrightarrow(10-i) e \leq(9.99-i) \Rightarrow e<1$.

\subsection{Minimal Cost Implementation: When Perfect isn't Good Enough}

Our focus on negative correlation in cost rather than correlation in occurrence distinguishes our approach from the Scientific and Classic Moral Hazard approaches. Distinguishing our 
model from the No-lying approach takes us from the issue of implementability to the issue of minimal cost implementation.

The most viable version of the No-lying approach is to posit the existence of "perfect evidence:" some package of evidence whose presentation is of infinite cost unless the agent has taken the target level of care (see Definition 3 for the precise meaning in the stochastic model). The evidence is "perfect" in the sense that no matter what the liability schedule $l(e)$, the agent's presentation of such evidence implies that he has taken the target level of care. ${ }^{27}$ If we associate presentation of this evidence with the meaning "I have taken target care," then it is as if the agent cannot lie in making this statement. The court can then implement care by imposing sufficient liability on any agent that fails to present this evidence.

The most obvious criticism of this conception of evidence is that perfect or even near perfect evidence is unlikely to exist. Though costly, forgery is almost always possible, and if sufficiently compensated in the liability schedule, likely to occur. The object of this section is to provide a somewhat more subtle criticism: we show that even if perfect evidence existed, we would not want to use it.

The object of civil process is not of course to seek and find perfect evidence of care, but to set incentives for (the proper amount of) care in the most efficient way possible. The problem with perfect evidence from this perspective is that it is wasteful. The reason is that large (including infinite) cost differences across care levels go typically hand in hand with large absolute costs for careful agents. Creating evidence cost differences in excess of what is necessary for implementation is expensive slack. Better to cut back on the evidence we require in a manner that makes it cheaper for all and yet still sufficiently more expensive for the careless. 
Suppose, for instance that we are implementing care by requiring agents to present an extensive battery of witness testimony, documents, and exhibits that, though quite expensive for careful agents is virtually impossible for the careless. To fix ideas, suppose this evidence costs the careful $\$ 10,000$ to compile and present, but costs the careless $\$ 100,000,000$. By setting a punishment for not presenting all witnesses, documents, and exhibits, we can create a difference in hearing payoffs of up to $\$ 99,990,000$. Assuming that the private cost of care is only $\$ 20,000$, this is more than enough to set incentives for care.

The perfect evidence story stops here: care is implementable. A more complete analysis considers whether the requisite cost differences are being created in the most efficient manner, and likely concludes not. We can always condition punishment on some portion of the perfect evidence package, say only the witness testimony and not the documents and exhibits. This would save money on evidence costs for careful agents and thus for society. Of course, it would also reduce evidence cost differences, but so long as these differences do not fall below $\$ 20,000$ we can still accomplish our purpose.

The remainder of this section formalizes and generalizes this argument. The first step in doing so is to realize that the preceding paragraph implicitly makes two assumptions. The first is that if we are willing to give up evidence costs differences, we can have lower absolute evidence costs. Though certainly an assumption, this is quite plausible: whatever we have the careful agent doing that is producing such stark cost differences, just have her do less of it. We might think of measuring evidence by its level of "detail." Requiring less detail means lower absolute costs and lower cost differences. 
The argument also relies on the assumption that the possible reductions are not too "chunky." If our only choices were, for example, differences of $\$ 99,990,000$ and $\$ 9$, then our argument would not go through. Perfect evidence would be the only implementing choice.

Both of these requirements are (more than) captured by the following definition:

Definition 1: Evidence is divisible for $(i, s)$ if arbitrarily close to any evidence with strictly positive cost under $(i, s)$ is evidence with strictly lower costs. Formally, $\forall e \in E$ such that $c_{i s}(e)>0, \forall$ open $^{28}$ neighborhoods $U(e)$ of $e, \exists e^{\prime} \in U(e)$ such that $c_{i s}\left(e^{\prime}\right)<c_{i s}(e)$. Evidence is divisible if it is divisible for all $(i, s)$.

We show that divisibility implies that in any solution to the court's problem, we never use perfect (or even nearly perfect) evidence for $(i, s)$. In particular, we never use evidence whose "minimal cost difference" exceeds some fixed finite bound related to the likelihood of the state and the private cost of the implemented action.

Definition 2: The minimal cost difference for circumstance $(i, s)$ and evidence $e$ is

$$
\min _{i^{\prime} s^{\prime} \neq i s}\left(c_{i^{\prime} s^{\prime}}(e)-c_{i s}(e)\right)
$$

Definition 3: Evidence $e$ is perfect for circumstance $(i, s)$ if the minimal cost difference for $(i, s)$ and $e$ is infinite.

Definition 4: Do-nothing evidence $0 \in E$ is evidence that is of minimal cost in all circumstances and also has the same cost across all circumstances. Formally, $\forall\left(i^{\prime}, s^{\prime}\right)$, $c_{i s^{\prime} s^{\prime}}(0)=\min _{e} c_{i^{\prime} s^{\prime}}(e)=F \geq 0$. 
We allow the uniform cost of do-nothing evidence to be positive to account for the possibility of fixed costs.

Proposition 2 [Upper Bound on Minimal Cost Difference in Minimal Cost Implementation]: Suppose that evidence is divisible and that do-nothing evidence exists. If the liability schedule $(l, e)$ implements action $i$ at minimal cost, then in every state $s$, the minimal cost difference for $(i, s)$ and evidence $e_{i s}$ does not exceed $\frac{1}{p_{s}}\left(a_{i}-a_{1}\right)$. In particular, perfect evidence is never used.

Example 2 [Application to Section 2]: In Section 2's example, the minimal cost evidence for implementing any $i \geq 2$ is hardly perfect. Indeed, the minimal cost difference for $i<10$ and evidence $e_{i}=1$ is negative,

$$
\min c_{i^{\prime}}\left(e_{i}\right)-c_{i}\left(e_{i}\right)=(11-10) e_{i}-(11-i) e_{i}=(i-10) e_{i}=i-10,
$$

meaning that not only is $i$ 's evidence possible for other actions, but $i$ is not even the most efficient at presenting it. And even if it is action $i=10$ that is implemented, so that the implemented action's cost advantage over all other actions can be made arbitrarily large by increasing $e_{10}$ to infinity, the optimally chosen advantage is quite modest, even viz. our established bound: the minimal cost difference is $(11-9) e_{10}-(11-10) e_{10}=e_{10}=1$ which is less than $a_{10}-a_{1}=9$ and certainly less than infinity.

\subsection{Which Action to Implement: Getting More for Less}

In this last subsection of our general discussion, we distinguish our model from those that employ reduced form trials. As noted in the introduction, those models imply that higher levels 
of care are more expensive to implement and so the second best level of care is less than the first. In Section 2, we presented an example of our model in which the second best exceeded the first. In this section we show that what happened in that example is not coincidental but reflective a general tendency. We emphasize that our different conclusion is a direct result of the fact that we explicitly model the trial process.

\subsubsection{Focusing on the Slope of the Minimal Cost of Implementation Schedule}

The first step is to understand the relationship between the slope of $C_{i}$ and the relative size of the first and second best. ${ }^{29}$ The basic idea, as embodied in the following observation, is straightforward. When implementation costs are downward sloping, both first best costs $a_{i}+h_{i}$ and implementation costs $C_{i}$ are larger "to the right of" the first best level. The only complications are the following: 1) the cost of implementing the lowest level of care is always zero and so implementation costs can only be downward sloping over $i \geq 2 ; 2$ ) in a discreet model such as this, the cost of implementation may not fall fast enough to conclude that the second best is strictly larger than the first; 3 ) some actions, including the first best, may not be implementable; 4) given the possibility of ties, there may be more than one first or second best level of care. Observation 1 and its preliminary definitions account for these complications.

Action $i$ is (a) first best if it minimizes the sum $a_{i}+h_{i}$ of the cost of precaution and the cost of accidents. Action $i$ is first best implementable if it minimizes $a_{i}+h_{i}$ among all actions that are implementable. Obviously, these two concepts differ only when a first best action is not implementable. Action $i$ is (a) second best if it minimizes $a_{i}+h_{i}+C_{i}$ and so also accounts for the minimal cost of implementation (recall that $C_{i}=\infty$ if $i$ is not implementable). 
Observation 1: Suppose that implementation costs are decreasing across all implementable actions greater than the $i=1$ : i.e. whenever $i$ and $2 \leq i^{\prime}<i$ are implementable, $C_{i^{\prime}}>C_{i}$. Then if $i^{S B} \geq 2$ is second best and $i^{F B I}$ is first best implementable, it must be that $i^{S B} \geq i^{F B I}$.

Example 3 [Application to Section 2]: Recall that in Section 2's example, $C_{i}$ was zero at $i=1$ and decreasing with a slope of -1 over $2 \leq i \leq 10$. Recall also that while the first best level of care was $i=5$, the second best level of care was $i=10$.

\subsubsection{Decomposing the Change in Implementation Costs}

The next step is to identify a downward sloping "tendency" in $C_{i}$. We formalize this tendency by first decomposing the slope of $C_{i}$ into two components and then showing that while one component is ambiguous in sign the other is always strictly negative, no matter what the functional form of evidence costs.

Observation 2: Suppose that liability per evidence schedule $(l, e)$ implements action $i \geq 2$ at minimal cost while $\left(l^{\prime}, e^{\prime}\right)$ implements some lower action $i^{\prime}<i$ at minimal cost. ${ }^{30}$ Then the net increase in implementation costs caused by increasing the implemented action from $i^{\prime}$ to $i$, namely $C_{i}-C_{i^{\prime}}=\sum_{s=1}^{S} p_{s} c_{i s}\left(e_{i s}\right)-\sum_{s=1}^{S} p_{s} c_{i^{\prime} s}\left(e_{i^{\prime} s}^{\prime}\right)$, may be additively decomposed ${ }^{31}$ into:

1) the change in presenter effect, $\sum_{s=1}^{S} p_{s}\left(c_{i s}\left(e_{i s}\right)-c_{i^{\prime} s}\left(e_{i s}\right)\right)$, and

2) the change in case effect, $\sum_{s=1}^{S} p_{s}\left(c_{i^{\prime} s}\left(e_{i s}\right)-c_{i^{\prime} s}\left(e_{i s}^{\prime}\right)\right)$.

The decomposition says that two things happen when we decrease the level of care that we are implementing from $i$ to $i^{\prime}$. First, fixing the evidence plan actually employed, we lower the 
action that is actually presenting the evidence. This corresponds to the change in presenter effect. Secondly, we shift the evidence per liability schedule from one that minimizes costs in implementing $i$ to one that minimizes costs in implementing $i^{\prime}$. This will generally entail a change in the evidence plan actually employed by the agent. The resulting change in expected costs, fixing the action taken at $i^{\prime}$, is the change in case effect.

\subsubsection{The Change in Presenter Effect is Always Negative}

The third step is to show that the change in presenter effect is unambiguously negative with no assumptions on evidence costs.

Proposition 3: The change-in-presenter effect $\sum_{s=1}^{S} p_{s}\left(c_{i s}\left(e_{i s}\right)-c_{i^{\prime} s}\left(e_{i s}\right)\right)$ is strictly negative.

The intuition is as follows. In order to implement $i$ over the lower $i^{\prime}$ we must be rewarding the agent with higher expected hearing payoffs if he takes action $i$ instead of the less privately costly action $i^{\prime}$. A fortiori the agent must do at least as well if he obediently takes action $i$ and honestly presents $e_{i s}$ in each state $s$, than if he disobediently takes action $i^{\prime}$ and then dishonestly mimics an obedient agent at the hearing: i.e., presents $e_{i s}$ (as opposed to $e_{i^{\prime} s}$ ) in each state. (There may be even better ways for the disobedient agent to be dishonest at the hearing.) Since private costs are strictly higher for the obedient and honest agent, it must be that the obedient and honest agent's expected hearing payoffs are strictly higher than those of the disobedient and dishonest agent. Since both agents present the same evidence in each state they obtain the same liability in each state. Thus the only way the obedient and honest agent's expected hearing payoffs can be higher is if his expected evidence costs are strictly lower. Note that we cannot conclude that his evidence costs are strictly lower in any given state. 
Example 4 [Application to Section 2]: In the Section 2's example, the change in presenter effect is simply equal to the additive inverse of the evidence that $i$ presents,

$$
c_{i}\left(e_{i}\right)-c_{i-1}\left(e_{i}\right)=\left((11-i) e_{i}-(11-(i-1)) e_{i}\right)=-e_{i}=-1 \text {. }
$$

\subsubsection{The Change in Case Effect is Ambiguous}

In general the change in case effect may go in either direction. We start with a simple and natural example where the effect is negative, thus reinforcing the change in presenter effect. Then we show that it can also be positive and in fact will be whenever evidence costs satisfy a special version of the single-crossing property.

Example 5 [Strictly Negative Change in Case Effect]: We have three actions 1, 2, and 3 with private costs 0,4 , and 10 respectively. Further we have two pieces of evidence. The first piece of evidence $\underline{e}$ costs $\$ 20,10$, and 0 for actions 1,2 and 3 respectively. The second piece of evidence $\bar{e}$ is more expensive for all actions, costing $\$ 25,20$, and 15 for actions 1,2 and 3 respectively. Note that each piece of evidence is less expensive for actions with higher private costs. Thus evidence costs are ordered "normally." However, evidence costs do not satisfy a single crossing property.

Now, action 3 can be implemented with the lowest cost evidence $\underline{e}$ : if the agent must present $\underline{e}$ in order to avoid some large amount of liability, then action 3's total payoffs of $-10-0=-10$ are larger than both action 1's, $-0-20=-20$ and action 2's, $-4-10=-14$. Hence, the minimal cost implementation of action 3 requires that action 3 present evidence $\underline{e}$.

Action 2, however, cannot be implemented with the lower cost evidence $\underline{e}$. For suppose that we were implementing action 2 with a liability schedule in which action 2 presents $\underline{e}$. 
Then action 3 can always present $\underline{e}$ also. This will give her the same liability as action 2 with $\$ 10$ less in evidence costs. Thus action 3's hearing payoffs, from whatever evidence she is called on to produce, must be at least $\$ 10$ more than action 2's. Since her private costs are only $\$ 6$ more, action 2 is not implemented.

On the other hand, action 2 is implemented if all actions present the more expensive evidence $\bar{e}$. Then action 2's total payoffs are $-4-20=-24$, which exceeds both action 1's payoffs, $-0-25=-25$ and action 3 's payoffs, $-10-15=-25$. Thus the minimal cost implementation of action 2 requires that action 2 present evidence $\bar{e}$.

Thus the evidence $\bar{e}$ used to implement action 2 at minimal cost is more costly (for all actions, specifically for action 2 herself) than the evidence $\underline{e}$ used to implement action 3 at minimal cost. We conclude that the change in case effect as between actions 2 and 3 is strictly negative.

Thinking about Example 5 in more general terms will illuminate why the change in case effect will always be non-negative when evidence costs satisfy a single-crossing property, which is the content of the next formal result. The simplicity of the intuition may convince the reader, as it has us, that imposing the single crossing property to sign the change in case effect is arbitrary and uninteresting - and that the best attitude toward the sign of the change in case effect is agnostic.

If we lower the action that we are implementing from $i$ to $i^{\prime}<i$, we must not increase the expected evidence cost difference $\sum_{s=1}^{S} p_{s}\left(c_{i^{\prime} s}\left(e_{s}\right)-c_{i s}\left(e_{s}\right)\right)$ for the evidence plan $e_{s}$ actually employed (before by $i$, now by $i^{\prime}$ ). Why? Because in implementing $i$ we had to insure that the agent could not gain by choosing $i^{\prime}$ and then presenting $i$ 's evidence in each state. Since this disobedience would net the same liability as obedience, it had to be that evidence cost 
differences were not exceeded by private cost differences: $\sum_{s=1}^{S} p_{s}\left(c_{i^{\prime} s}\left(e_{s}\right)-c_{i s}\left(e_{s}\right)\right) \geq a_{i}-a_{i^{\prime}}$. The point is that if we strictly increase the left hand side of this inequality in our new implementation of $i^{\prime}$, the agent will have a strict incentive to choose $i$ over $i^{\prime}$ for we will not satisfy the requisite $\sum_{s=1}^{S} p_{s}\left(c_{i s}\left(e_{s}\right)-c_{i^{\prime}}\left(e_{s}\right)\right) \geq a_{i^{\prime}}-a_{i}$.

Now in Example 5, the difference $\sum_{s=1}^{s} p_{s}\left(c_{i^{\prime} s}\left(e_{s}\right)-c_{i s}\left(e_{s}\right)\right)$ was strictly greater than private costs differences in implementing action 3 (it was 10 for $i^{\prime}=2$ ). Thus we had to strictly reduce it in changing target care from 3 to 2 . Given the parameter values, reducing this difference viz. actions 2 and 3 required increasing evidence costs for action 2, by switching from $\underline{e}$ to $\bar{e}$. Since $\bar{e}$ was more expensive for 2 than $\underline{e}$, the change in case effect was there negative.

Of course we could always rule this out by arbitrarily assuming that expected cost differences $\sum_{s=1}^{S} p_{s}\left(c_{i s}\left(e_{s}\right)-c_{i s}\left(e_{s}\right)\right)$ always move in tandem with $i^{\prime}$ 's expected costs $\sum_{s=1}^{S} p_{s} c_{i^{\prime} s}\left(e_{s}\right)$. This is of course a version of the single crossing property, here with respect to the ordering on evidence per state plans $e \in E^{S}$ induced by the cost function $\sum_{s=1}^{S} p_{s} c_{i^{\prime} s}\left(e_{s}\right)$ :

Definition 5: Evidence costs satisfy the single crossing property over evidence plans if for all implementable $i \geq 2$ and all implementable $i^{\prime}<i$,

$$
\sum_{s=1}^{S} p_{s}\left(c_{i^{\prime} s}\left(e_{s}\right)-c_{i s}\left(e_{s}\right)\right) \geq \sum_{s=1}^{S} p_{s}\left(c_{i^{\prime} s}\left(e_{s}^{\prime}\right)-c_{i s}\left(e_{s}^{\prime}\right)\right) \text { implies } \sum_{s=1}^{S} p_{s} c_{i^{\prime} s}\left(e_{s}\right) \geq \sum_{s=1}^{S} p_{s} c_{i^{\prime} s}\left(e_{s}^{\prime}\right) .
$$

Proposition 4: If evidence costs satisfy the single crossing property over evidence plans, as defined in Definition 5, then the change in case effect is non-negative. 
Example 6 [Application to Section 2]: Returning (for the last time) to the example in Section 2, we see that it does satisfy the single-crossing property for evidence plans (which in this deterministic example are scalars). Employing the contrapositive of Definition 5, $(11-i) e=c_{i-1}(e)>c_{i-1}\left(e^{\prime}\right)=(11-i) e^{\prime}$ implies $e>e^{\prime}$ which implies $e=c_{i-1}(e)-c_{i}(e)>c_{i-1}\left(e^{\prime}\right)-c_{i}\left(e^{\prime}\right)=e^{\prime}$. But this guarantees only that the change in case in nonnegative, not that it is positive. Indeed, it is zero in that example since the minimal cost liability per evidence schedule does not change across implemented actions.

\section{Special Case: Deterministic, Multiplicatively Separable Evidence}

\section{Costs}

Obtaining sharper results than those presented in Section 3 requires placing functional form assumptions on evidence costs. This is precisely the plan in this section and the following. Here we assume that evidence costs are a deterministic function of action and are multiplicatively separable across action and evidence. That is, we assume that for all $i=1, \ldots, I, c_{i}(e)=\varsigma_{i} c(e)$, where $\zeta_{i} \in \Re_{++}$is action $i$ 's (evidence) cost coefficient and $c$ maps $E$ onto ${ }^{32} \mathfrak{R}_{+}$. Note that we do not assume that $\varsigma_{i}$ is decreasing in $i$.

This class of evidence costs contains the simple example from Section 2; there $E=\mathfrak{R}_{+}$, $c(e)=e$ and $\varsigma_{i}=11-i$. Moreover, it is the only functional form for which it makes sense to speak of the "relative increase" and "relative convexity ${ }^{33}$ " of evidence costs in actions without mentioning which evidence $e \in E$ is being presented. In particular,

$$
\frac{c_{i}(e)-c_{i-1}(e)}{c_{i-1}(e)}=\frac{\left(\zeta_{i}-\varsigma_{i-1}\right) c(e)}{\zeta_{i-1} c(e)}=\frac{\left(\varsigma_{i}-\varsigma_{i-1}\right)}{\zeta_{i-1}} \equiv \frac{\Delta \varsigma_{i}}{\varsigma_{i-1}} \quad \text { all } i=2, \ldots, I
$$

and 


$$
\frac{\left(c_{i}(e)-c_{i-1}(e)\right)-\left(c_{i-1}(e)-c_{i-2}(e)\right)}{c_{i-1}(e)-c_{i-2}(e)}=\frac{\left(\varsigma_{i}-\varsigma_{i-1}\right) c(e)-\left(\varsigma_{i-1}-\varsigma_{i-2}\right) c(e)}{\left(\varsigma_{i-1}-\varsigma_{i-2}\right) c(e)}=\frac{\left(\varsigma_{i}-\varsigma_{i-1}\right)-\left(\varsigma_{i-1}-\varsigma_{i-2}\right)}{\left(\zeta_{i-1}-\varsigma_{i-2}\right)} \equiv \frac{\Delta^{2} \varsigma_{i}}{\Delta \varsigma_{i-1}}, \quad \text { all } i=3, \ldots, I
$$

The uniformity of these parameters across $E$ allows us to give sharp analytical and geometric answers to the three questions posed by the principal's overall problem. In particular, we show how the slope of the minimal cost of implementation schedule turns on the relationship between the rate at which private costs increase and the relative convexities of $a_{i}$ in $\zeta_{i}$.

\subsection{Implementability}

Figure 4 shows a plot of the points $\left(a_{i}, \varsigma_{i}\right)$ for $i=1, \ldots, I$. Thus it shows the functional relationship between evidence costs and private costs. The following proposition says that action $i$ is implementable if and only if $\left(a_{i}, \varsigma_{i}\right)$ is on the southwestern frontier of this set of points. Thus in that figure, $i^{\prime}$ is implementable but $i^{\prime \prime}$ is not.

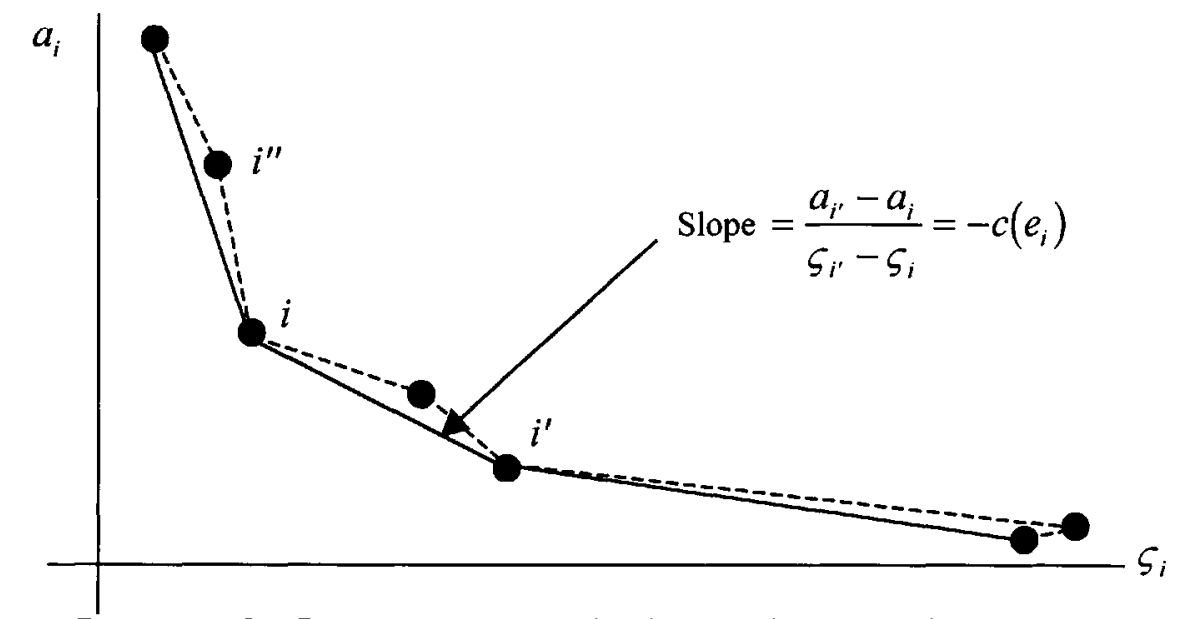

Figure 4: Diagram for Deterministic Multiplicatively Separable Evidence Costs

Proposition 5: Action $i$ is implementable IF AND ONLY IF $i$ minimizes $a_{i}+\alpha \varsigma_{i}$ for some $\alpha \geq 0$. 
Corollary 3: All actions are implementable IF AND ONLY IF evidence costs $\varsigma_{\text {, }}$ are strictly decreasing and convex ${ }^{34}$ in private costs $a_{i}$.

\subsection{Minimal Cost Implementation}

The next proposition concerns the minimal cost implementation of implementable action $i$. Graphically it tells us that the evidence $e_{i}$ presented by $i$ in any minimal cost implementation is set so that $c\left(e_{i}\right)$ equals the absolute value of the slope from $\left(\varsigma_{i}, a_{i}\right)$ to $\left(\varsigma_{i^{\prime}}, a_{i^{\prime}}\right)$, where $i^{\prime}<i$ is the next lowest implementable action. (Since $i=1$ is always implementable, there will always be such an $i^{\prime}$.) Thus in Figure 4 evidence presented in the minimal cost implementation of action $i$ is such that $c\left(e_{i}\right)=-\frac{a_{r^{\prime}}-a_{i}}{c_{i^{\prime}}-c_{i}}$.

Proposition 6: If action $i \geq 2$ is implementable and $i^{\prime}<i$ is the next lowest ${ }^{35}$ implementable action (which always exists), then $\left(\varsigma_{i}<\varsigma_{i^{\prime}}\right.$ and) $c\left(e_{i}\right)=-\frac{a_{r^{\prime}}-a_{i}}{c_{i^{\prime}}-c_{i}}$ in any minimal cost implementation of $i$.

\subsection{Which Action to Implement}

We can use Proposition 6 to understand graphically whether the minimal cost of implementation rises or falls as we move through the implementable actions (we ignore actions that are not implementable). As we move to the next highest implementable action (skipping any non-implementable actions in between), we move in a northwesterly direction up the southwestern frontier drawn with a solid line in Figure 4. Recall from Section 3.3.2 that two subsidiary changes determine the overall change in the minimal cost of implementation. First, $\varsigma_{i}$ is decreasing: since both the action level we are at and the action level we are moving to are 
on the southwestern frontier, the line between them must have negative slope in our Figure 4 and so $\varsigma_{i}$ must be decreasing. This decrease in $\zeta_{i}$ corresponds to the change in presenter effect identified in Section 3.3.2. It is strictly negative, consistent with Proposition 3. Note that we have not assumed that $\varsigma_{i}$ decreases $i$ : Proposition 3 implies that it must decrease in the move to the next highest implementable action.

The other subsidiary change in moving to the next highest implementable action level is that the slope of the frontier becomes steeper (more negative). That the slope steepens is again a consequence of Proposition 5. The implication, that evidence presented increases, is a consequence of Proposition 6. This is called the change in case effect in Section 3.3.2. Though this effect is generally ambiguous, it is always non-negative with this functional form for evidence costs. This is consistent with Proposition 4, which says that the change in case effect is non-negative when evidence costs satisfy a single crossing property. Since $\varsigma_{i}$ increases across implementable actions, this requisite single crossing property is always satisfied for this class of evidence costs.

Thus in this example, the two effects, the change in presenter and change in case work at cross purposes. Which will win out? As is clear from Figure 4, this depends on how fast the slope of the frontier increases as we decrease $\varsigma_{i}$ across implementable actions. If the frontier is near linear, then the change in presenter effect will dominate and higher levels of action will be less costly to enforce. This is precisely what happened in the example in Section 2: there we had a linear frontier since $a_{i}-a_{i-1}=i-(i-1)=-\left(\varsigma_{i}-\varsigma_{i-1}\right)$. Notice that the change in case effect would still be zero in this example after applying any affine transformation to either or both private costs and evidence cost coefficients. On the other hand, if the frontier is quite convex, then the change in case effect will dominate. 
What then makes the frontier more convex? The more convex are private costs and evidence costs in actions, the more convex the frontier. As we increase actions among those that are implementable, thus moving left in Figure 4, we want the decrease in evidence costs to slow and the increase in private costs to speed up. ${ }^{36}$

These remarks are formalized in the following proposition. To state the proposition concisely let $\{1, \ldots, K\}$ denote the subset of implementable actions numbered, like $\{1, \ldots, I\}$, in order of increasing private costs. Thus to obtain $\{1, \ldots, K\}$ we pull out all non-implementable actions from $\{1, \ldots, I\}$ and then renumber the remainder consecutively.

Proposition 7: For all implementable actions $k \geq 3$, the following describes the change in the minimal cost of implementation:

$$
C_{k}-C_{k-1}=\underbrace{-\Delta a_{k}}_{\text {change in presenter effect }}+\underbrace{\alpha_{k}\left(\frac{\Delta^{2} a_{k}}{\Delta a_{k-1}}+\frac{\Delta^{2} \varsigma_{k}}{-\Delta \zeta_{k-1}}\right)}_{\text {change in case effect }},
$$

where $\alpha_{k}=\frac{\zeta_{k-1} \Delta a_{k-1}}{-\Delta \zeta_{k}}>0$. The change in presenter effect $-\Delta a_{k}$ is strictly negative and the change in case effect $\alpha_{k}\left(\frac{\Delta^{2} a_{k}}{\Delta a_{k-1}}+\frac{\Delta^{2} \varsigma_{k}}{-\Delta \zeta_{k-1}}\right)$ is non-negative.

We conclude from (13) that the change in presenter effect is significant when private costs increase steeply across implementable actions (i.e. when $\Delta a_{k}$ is large). The change in case effect is significant when private costs are very convex in evidence costs (e.g., when private 
costs increase at an increasing pace so that $\frac{\Delta^{2} a_{k}}{\Delta a_{k-1}}$ is large positive, and evidence costs decrease at a decreasing pace so that $\frac{\Delta^{2} \varsigma_{k}}{-\Delta \zeta_{k-1}}$ is also large positive). ${ }^{37}$

\section{Special Case: Stochastic Multipicatively Separable Evidence Costs}

In this section we study implementability and minimal cost implementation with stochastic multiplicatively separable evidence costs. First, we provide a necessary and sufficient condition for implementability in a stochastic framework (Proposition 1 is only necessary). Second, we characterize the minimal cost liability schedule for a given level of care. We have seen that with deterministic costs of any form, the minimal cost liability schedule may take a very simple form with all actions presenting the same evidence. ${ }^{38}$ This is not generally true with stochastic costs. However, we are able to show under the functional form assumptions in this section that the number of distinct cases employed in any minimal cost implementation will not exceed the number of actions in the underlying activity. This is the most interesting implication of the section's final result, Proposition 9.

Similar to the previous section, let evidence costs be of the form $c_{i s}(e)=\zeta_{\text {is }} c(e)$, where $c(e)$ is onto $\mathfrak{R}_{+}$and all $\varsigma_{\text {is }}$ are positive integers. ${ }^{39}$ Letting $J$ be the largest integer among the (finitely many) $\varsigma_{i s}$, define for each action $i$ its distribution $P_{i}=\left(P_{i 1}, \ldots, P_{i J}\right) \geq 0$ over all possible values of evidence cost coefficients: $P_{i j}=\sum_{s: \zeta_{i s}=j} p_{s}, j=1, \ldots, J$. Granted, some integers $j$ may have no associated circumstance $(i, s)$, but it is convenient and harmless to include hearing constraints for these as well. Conversely, circumstances with the same cost coefficient must be treated identically at the hearing and so there is nothing lost in treating them like a single circumstance. Thus we may proceed as if action $i$ leads to evidence cost parameter $j$ with 
probability $P_{i j}$. We then test underlying activity incentives in the same way, but for the hearing constraint, we test each $j$ against all other $j^{\prime}$.

\subsection{Implementability}

LEMMA 2 in the appendix shows that hearing payoffs must be non-increasing and convex in $j$. This accounts for the prominence of second order stochastic dominance in the following necessary and sufficient condition for implementability.

A mixed action is a probability measure $\pi=\left(\pi_{1}, \ldots, \pi_{l}\right)^{\prime} \geq 0, \sum_{i=1}^{l} \pi_{i}=1$ over actions. Its private costs are $\sum_{i=1}^{l} \pi_{i} a_{i}$. Its cost distribution is the convex combination $\sum_{i=1}^{l} \pi_{i} P_{i}$. Write $F_{i}=\left(F_{i 1}, \ldots, F_{i J}\right)$ for the cumulative distribution of $P_{i}$. Recall that $P_{i}$ first order stochastically dominates $P_{i^{\prime}}$ if $F_{i} \leq F_{i^{\prime}}$. Roughly, $P_{i}$ first order stochastically dominates $P_{i^{\prime}}$ when it puts more weight on higher values of $j$. Write $G_{i}$ for the double cumulative of $P_{i}$, i.e.

$G_{i j}=\sum_{j^{\prime}=1}^{j} \sum_{j^{\prime \prime}=1}^{j^{\prime}} P_{i^{\prime \prime}}=\sum_{j^{\prime}=1}^{j} F_{i j^{\prime}}$. The distribution $P_{i}$ second order stochastically dominates $P_{i^{\prime}}$ if $G_{i} \leq G_{i^{\prime}}$. Thus, first order stochastic dominance implies second. Roughly, $P_{i}$ second order stochastically dominates $P_{i^{\prime}}$ when it is both more concentrated and puts more weight on higher values.

Proposition 8: Action $i$ is implementable IF AND ONLY IF its cost distribution $P_{i}$ does not second order stochastically dominate the cost distribution of a mixed action with strictly lower private costs.

Remark 2 [Comparison to Conditions in Hermalin and Katz (1991)]: Compare this condition to that for implementation in the classic moral hazard model, as derived in Hermalin and Katz 
(1991): action $i$ is implementable if and only if its signal distribution does not first order stochastically dominate the signal distribution of a mixed action with the same private costs.

Corollary 4: Action $i$ is implementable ONLY IF its cost distribution $P_{i}$ does not first order stochastically dominate the cost distribution of a mixed action with strictly lower private costs.

Corollary 5: Action $i$ is implementable ONLY IF its cost distribution $P_{i}$ does not first order stochastically dominate the cost distribution of an action with strictly lower private costs.

\subsection{Minimal Cost Implementation}

The following result establishes that the optimal liability per evidence schedule for implementing a given action $i$ is a monotonic step function with $I$ steps, as depicted in Figure 5. In particular, we will use no more than $I$ different pieces of evidence in implementing each action $i$. Interestingly, the $i^{\prime}$ th evidence will have no particular relationship to action $i^{\prime}$.

Proposition 9: For some (generically, all) liability schedules $(l, e)$ implementing action $i$ at minimal cost, there exists an $I$-cell interval partition of $\{1, \ldots, J\}, j_{0} \equiv 1 \leq j_{1} \leq \ldots \leq j_{I}$ with the following property: for each cell $\left[j_{i^{\prime}-1}, j_{i^{\prime}}\right]$ and each $j, j^{\prime}$ therein with $P_{i j}, P_{i j^{\prime}}>0$, we have $l_{j}=l_{j^{\prime}} \equiv l^{i^{\prime}}$ and $c\left(e_{j}\right)=c\left(e_{j^{\prime}}\right) \equiv c^{i^{\prime}}$. Moreover, $l^{i^{\prime}}$ is non increasing in $i^{\prime}, c^{i^{\prime}}$ is non decreasing in $i^{\prime}$ and $c^{1}=0$. 


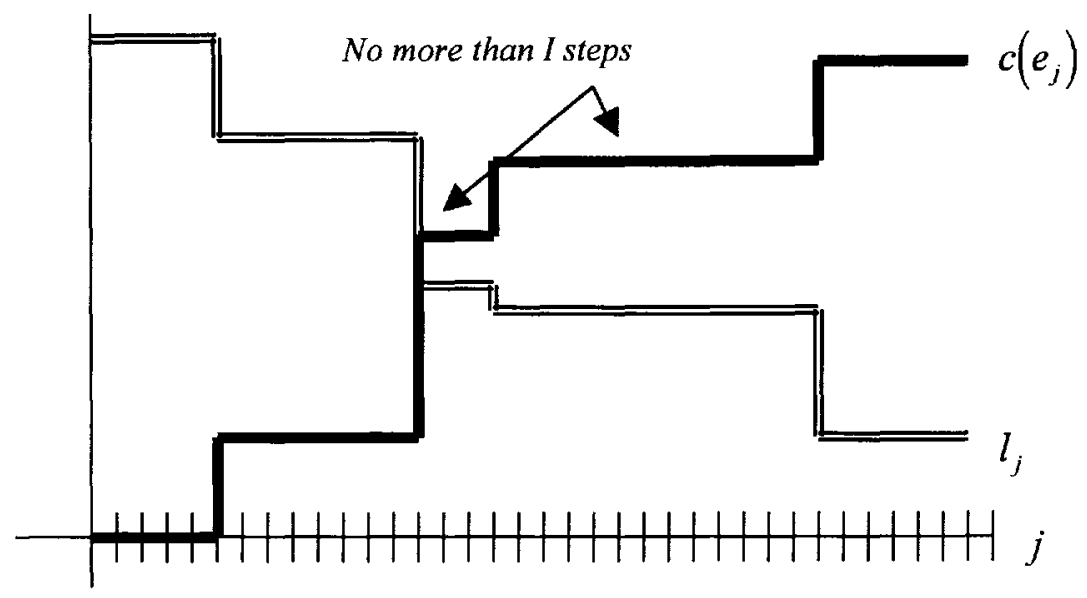

Figure 5: The I-step Liability per Evidence Schedule Implementing $i$ at Minimal Cost

\section{Conclusion}

Great progress has been made in the last ten years in understanding the economics of civil litigation. Our understanding of care choice, filing behavior, trial preparation, and pre-trial negotiation have all moved forward at rapid pace. The glaring exception to this general advancement has been our understanding of what actually happens in the court room-at the terminal node, as it were, of the litigation game tree. Since all other studied aspects occur "in the shadow" of prospective courtroom events, it seems imperative to remedy this omission.

The object of this paper has been to take an initial step in that direction. Thus, we have presented a model of evidence production in single agent mandatory, periodic hearings, (leaving multiple agents and privately instigated suits to another paper (Sanchirico (1997)). The model is by no means a complete picture of court room process, but it makes several crucial conceptual points, which taken together represent a shift in the way evidence has been viewed both in law and in economics. First, in a world in which agents can and will lie, evidence is only of use in inspiring care on the street to the extent that the exercise of care lowers presentation cost in the court room. Second, this being so, enforcing higher levels of care turns agents into lower cost 
evidence producers and may reduce the cost of implementation. Since this in turn implies that the second best level of care will exceed the first, it calls into serious question the given interpretation of recent claims that the tort system "overdeters" relative to first best levels. Third, the best evidence is not necessarily the most "conclusive." Presentation cost differences are necessary to implement care, but the absolute level of evidence costs for the implemented level of care is also an important factor. Thus, in contrast to proffered interpretations of the predominant "No-lying" models of evidence, "perfect evidence"-evidence that can not falsified -may be inferior to relatively forgeable evidence that costs significantly less when genuine.

\section{APPENDICES}

Proposition 10 [Equivalence of Natural and Direct Bifurcated Problems]: If $i,\left(e_{1}, \ldots, e_{S}\right)$ and $l(e)$ satisfy (2) and (3), then we can find $(l, e)$ with $e_{i s}=e_{s}$ for all $s=1, \ldots, S$ and $l_{i^{\prime} s^{\prime}}=l\left(e_{i^{\prime} s^{\prime}}\right)$ for all $\left(i^{\prime}, s^{\prime}\right)$ such that $i$ and $(l, e)$ satisfy (5) and (6). Conversely, if $i$ and $(l, e)$ satisfy (5) and $(6)$, then we can find a function $l(e)$ with $l_{i^{\prime} s^{\prime}}=l\left(e_{i^{\prime} s^{\prime}}\right)$ for all $\left(i^{\prime}, s^{\prime}\right)$ such that $i,\left(e_{i 1}, \ldots, e_{i S}\right)$ and l(e) satisfy (2) and (3).

Proof: The proof is a combination of familiar arguments, which we recite solely for the reader's convenience. If $\left(i ; e_{1}, \ldots, e_{s}\right)$ satisfies $(2)$ and (3) for $l(e)$, then set $(l, e)$ as follows: For $i$ and any $s$ set $e_{i s}=e_{s}$ and $l_{i s}=l\left(e_{i s}\right)=l\left(e_{s}\right)$. For all $i^{\prime} \neq i$ and any $s$, set $e_{i^{\prime} s}$ to any $e$ minimizing $l(e)+c_{i s^{\prime} s}(e)$ and set $l_{i^{\prime} s}=l\left(e_{i^{\prime} s}\right)$. Then for all $\left(i^{\prime}, s^{\prime}\right), l_{i^{\prime} s^{\prime}}+c_{i^{\prime} s^{\prime}}\left(e_{i^{\prime} s^{\prime}}\right)=\min _{e}\left[l(e)+c_{i^{\prime} s^{\prime}}(e)\right]$. From this and (2) it follows that $i$ satisfies the hearing and underlying activity constraints with the 
constructed $(l, e)$. Conversely, if $i$ and $(l, e)=\left(l_{11}, \ldots, l_{I S} ; e_{11}, \ldots, e_{I S}\right)$ satisfy the direct problem's constraints, construct $l(e)$ as follows: For any $K$, set

$$
l(e)=\left\{\begin{array}{ll}
l_{i s^{\prime}} & \text { if } e=e_{i s^{\prime}} \\
K & \text { if } e \text { is not equal to any } e_{i^{\prime} s^{\prime}}
\end{array} .\right.
$$

This assignment of $l$ to $e$ is unique since the hearing constraint guarantees that $l_{i^{\prime} s^{\prime}}=l_{i^{\prime \prime} s^{\prime \prime}}$ whenever $e_{i^{\prime} s^{\prime}}=e_{i^{\prime \prime} s^{\prime \prime}}$. If $K$ is large enough, then setting $e$ to a value that equals no $e_{i^{\prime} s^{\prime}}$ is never optimal for the agent at the hearing in any circumstance. Hence, the only relevant alternatives for the agent in circumstance $\left(i^{\prime}, s^{\prime}\right)$ are those assigned to other circumstances. Thus $e_{i^{\prime}}$ minimizes $l(e)+c_{i^{\prime} s}(e)$ for every $i^{\prime}$ and $s$, yielding (3). It is then clear that $i$ satisfies (2) against $l(e)$.

Proof of Proposition 1: Consider any alternative action $i^{\prime}$. There must be no advantage to the agent from taking action $i^{\prime}$ and then, in every state, presenting the evidence that she would have presented had she taken action $i$. From the underlying activity constraint

$$
-a_{i}+\sum_{s} p_{s}\left(-l_{i s}-c_{i s}\left(e_{i s}\right)\right) \geq-a_{i^{\prime}}+\sum_{s} p_{s}\left(-l_{i^{\prime} s}-c_{i^{\prime} s}\left(e_{i^{\prime} s}\right)\right)
$$

From the hearing constraint:

$$
\forall s=1, \ldots, S \quad-l_{i^{\prime} s}-c_{i^{\prime} s}\left(e_{i^{\prime} s}\right) \geq-l_{i s}-c_{i^{\prime} s}\left(e_{i s}\right)
$$

Substituting (16) into the right hand side of (5) yields

$$
-a_{i}+\sum_{s} p_{s}\left(-l_{i s}-c_{i s}\left(e_{i s}\right)\right) \geq-a_{i^{\prime}}+\sum_{s} p_{s}\left(-l_{i s}-c_{i^{\prime} s}\left(e_{i s}\right)\right) .
$$

Canceling the $l_{i s}$ terms and rearranging yields the condition.

Proof of Corollary 2: Having all actions present $e$ and obtain zero liability will implement $i$. 
Proof of Proposition 2: Suppose, on the contrary, that $c_{i^{\prime} s^{\prime}}\left(e_{i s}\right)-c_{i s}\left(e_{i s}\right)>\frac{a_{i}-a_{1}}{p_{s}}$ for all $\left(i^{\prime}, s^{\prime}\right) \neq(i, s)$. By divisibility there exists some other $e^{\prime}$ that has strictly lower costs for $(i, s)$ $\left(c_{i s}\left(e^{\prime}\right)<c_{i s}\left(e_{i s}\right)\right)$ and still satisfies $c_{i s^{\prime}}\left(e^{\prime}\right)-c_{i s}\left(e^{\prime}\right)>\frac{a_{i}-a_{1}}{p_{s}}, \forall\left(i^{\prime}, s^{\prime}\right) \neq(i, s)$. We will show that we can implement $i$ with a liability schedule whose costs are strictly lower than $(l, e)$ 's.

Set $e_{i^{\prime} s^{\prime}}=0 \in E$ and $l_{i^{\prime} s^{\prime}}=-F \in \mathfrak{R}$ for all $\left(i^{\prime} s^{\prime}\right) \neq(i, s)$ (including outcomes with the same action but different states, and vice versa). Set $e_{i s}=e^{\prime}$ and $l_{i s}=-\min _{i^{\prime} s^{\prime} \neq i s} c_{i^{\prime} s^{\prime}}\left(e^{\prime}\right)$. Note that the cost of this schedule is less than for $(l, e) p_{s} c_{i s}\left(e^{\prime}\right)+(S-1) F<p_{s} c_{i s}\left(e_{i s}\right)+(S-1) F \leq \sum_{s^{\prime}} p_{s^{\prime}} c_{i s^{\prime}}\left(e_{i s^{\prime}}\right)$.

Secondly, we claim that the schedule is incentive compatible at the hearing. We need to check only $(i, s)$ viz. its alternatives $\left(i^{\prime}, s^{\prime}\right)$. Hearing payoffs for $(i, s)$ are non-negative: $-l_{i s}-c_{i s}\left(e^{\prime}\right)=\min _{i^{\prime} s^{\prime} \neq i s} c_{i^{\prime} s^{\prime}}\left(e^{\prime}\right)-c_{i s}\left(e^{\prime}\right)>\frac{a_{i}-a_{1}}{p_{s}} \geq 0$. Since pretending to be any $\left(i^{\prime}, s^{\prime}\right)$ yields $(i, s)$ payoffs of $-(-F)-c_{i s}(0)=0,(i, s)$ has no incentive to lie. Conversely, pretending to be $(i, s)$ yields an alternative outcome $\left(i^{\prime}, s^{\prime}\right)$ payoffs $-l_{i s}-c_{i s^{\prime}}\left(e^{\prime}\right)=-\min _{i^{\prime \prime} s^{\prime \prime} \neq i s} c_{i^{\prime \prime} s^{\prime \prime}}\left(e^{\prime}\right)-c_{i^{\prime} s^{\prime}}\left(e^{\prime}\right) \leq 0$, while truth telling yields payoffs of $-(-F)-c_{i^{\prime} s^{\prime}}(0)=0$.

We complete the proof by showing that $(l, e)$ is incentive compatible in the underlying activity. For any $i^{\prime}$,

$$
\begin{aligned}
& -a_{i}+\sum_{s^{\prime}} p_{s^{\prime}}\left(-l_{i s^{\prime}}-c_{i s^{\prime}}\left(e_{i s^{\prime}}\right)\right) \\
= & -a_{i}+p_{s}\left(\min _{i^{\prime} s^{\prime} \neq i s} c_{i^{\prime} s^{\prime}}\left(e^{\prime}\right)-c_{i s}\left(e^{\prime}\right)\right) \\
\geq & -a_{i}+p_{s}\left(\frac{a_{i}-a_{1}}{p_{s}}\right) \\
= & -a_{i}+a_{i}-a_{1} \\
\geq & -a_{i^{\prime}}+0 \\
= & -a_{i^{\prime}}+\sum_{s^{\prime}} p_{s^{\prime}}\left(-l_{i^{\prime} s^{\prime}}-c_{i s^{\prime}}\left(e_{i i^{\prime} s^{\prime}}\right)\right) .
\end{aligned}
$$


Proof of Observation 1: Suppose that $i^{F B I}$ is first best implementable. Consider any lower level $2 \leq i^{\prime}<i^{F B I}$ of positive care. We will show that $i^{\prime}$ cannot be second best. If $i^{\prime}$ is not implementable, then it is certainly not the second best. If $i^{\prime}$ is implementable, then since $i^{F B I}$ is first best implementable, the sum of precaution and accident costs is lower at $i^{F B I}$ than at $i^{\prime}$ : $-a_{i^{F E I}}-h_{i^{F B I}} \geq-a_{i^{\prime}}-h_{i^{\prime}}$. Since $C_{i}$ is downward sloping, implementation costs are strictly lower at $i^{F B I}$. Thus $i^{F B I}$ has strictly lower total costs than $i^{\prime}$ and so $i^{\prime}$ cannot be second best. Proof of Proposition 3: Immediate from the last line of Proposition 1.

Proof of Proposition 4: Suppose that $i$ 's evidence plan, when $i$ is implemented, is $e=\left(e_{1}, \ldots, e_{s}\right)$. Suppose that $i^{\prime}<i$ 's evidence plan when $i^{\prime}$ is implemented is $e^{\prime}=\left(e_{1}^{\prime}, \ldots, e_{S}^{\prime}\right)$. When $i$ is implemented, the agent must have no incentive to choose $i$ ' and then present $i$ 's evidence. By the reasoning employed in the proof of Proposition 2, this means $a_{i}-a_{i^{\prime}} \leq \sum_{s=1}^{S} p_{s}\left(c_{i^{\prime}}\left(e_{s}\right)-c_{i s}\left(e_{s}\right)\right)$. Similarly, when $i^{\prime}$ is implemented, the agent must have no incentive to choose $i$ and present $i^{\prime}$ 's evidence. Therefore, $a_{i}-a_{i^{\prime}} \geq \sum_{s=1}^{S} p_{s}\left(c_{i^{\prime} s}\left(e_{s}^{\prime}\right)-c_{i s}\left(e_{s}^{\prime}\right)\right)$. Combining yields $\sum_{s=1}^{S} p_{s}\left(c_{i^{\prime} s}\left(e_{s}\right)-c_{i s}\left(e_{s}\right)\right) \geq \sum_{s=1}^{S} p_{s}\left(c_{i^{\prime} s}\left(e_{s}^{\prime}\right)-c_{i s}\left(e_{s}^{\prime}\right)\right)$, which by the single crossing property for evidence plans implies $\sum_{s=1}^{S} p_{s} c_{i^{\prime} s}\left(e_{s}\right) \geq \sum_{s=1}^{S} p_{s} c_{i^{\prime} s}\left(e_{s}^{\prime}\right)$ Proof of Proposition 5: By Corollary 2, $i$ is implementable, if and only if $\exists e \in E$ s.t. $\forall$ $i^{\prime}=1, \ldots, I,\left(\varsigma_{i^{\prime}}-\varsigma_{i}\right) c(e)=c_{i^{\prime}}(e)-c_{i}(e) \geq a_{i}-a_{i^{\prime}} \Leftrightarrow a_{i^{\prime}}+c(e) \varsigma_{i^{\prime}} \geq a_{i}+c(e) \varsigma_{i}$. The forward direction of the proposition then follows from $c$ 's non-negativity; the backward direction from the fact that $c$ is onto $\mathfrak{R}_{+}$ 
Proof of Proposition 6: Suppose that $(l, e)$ implements $i$. Then from the last line in Proposition $1,\left(\varsigma_{i^{\prime}}-\varsigma_{i}\right) c\left(e_{i}\right)=c_{i^{\prime}}\left(e_{i}\right)-c_{i}\left(e_{i}\right) \geq a_{i}-a_{i^{\prime}}>0$. Thus $\varsigma_{i^{\prime}}-\varsigma_{i}>0$ and $c\left(e_{i}\right) \geq-\frac{a_{i}-a_{i^{\prime}}}{\varsigma_{i}-\varsigma_{i^{\prime}}}$. Thus, no evidence schedule implementing $i$ can be cheaper than one with $c\left(e_{i}\right)=-\frac{a_{i}-a_{i}}{c_{i}-c_{i}}$.

It remains to show that we can implement $i$ with a schedule satisfying $c\left(e_{i}\right)=-\frac{a_{i}-a_{i}}{c_{i}-c_{i}}$.

Consider a schedule in which all $l_{i^{\prime \prime}}=0$ and all $e_{i^{\prime \prime}}$ equal some $\bar{e}$ satisfying $c(\bar{e})=-\frac{a_{r}-a_{l}}{c_{r^{\prime}}-c_{r^{\prime}}}$. This schedule is trivially incentive compatible at the hearing. Suppose, contra, that it is not incentive compatible in the underlying activity. Then from the underlying activity constraint, we can find

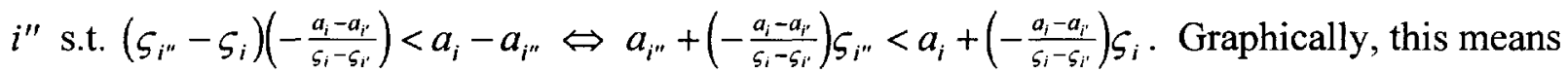
that $\left(\varsigma_{i^{\prime \prime}}, a_{i^{\prime \prime}}\right)$ lies strictly southwest of the line connecting $\left(\varsigma_{i}, a_{i}\right)$ and $\left(\varsigma_{i^{\prime}}, a_{i^{\prime}}\right)$. That this cannot be the case is easy to see graphically (but tedious algebraically). If $\left(\varsigma_{i^{\prime \prime}}, a_{i^{\prime \prime}}\right)$ is to the left of both $\left(\varsigma_{i}, a_{i}\right)$ and $\left(\varsigma_{i^{\prime}}, a_{i^{\prime}}\right)$ (i.e. $\left.\varsigma_{i^{\prime \prime}} \leq \varsigma_{i}<\varsigma_{i^{\prime}}\right)$, then $i$ cannot be implementable because it is not on the southwestern frontier, as required by Proposition 5. Similarly, if $\left(\varsigma_{i^{\prime \prime}}, a_{i^{\prime \prime}}\right)$ is to the right of both $\left(\varsigma_{i}, a_{i}\right)$ and $\left(\varsigma_{i^{\prime}}, a_{i^{\prime}}\right)$, then $i^{\prime}$ could not be implementable. If $\left(\varsigma_{i^{\prime \prime}}, a_{i^{\prime \prime}}\right)$ is horizontally between $\left(\varsigma_{i}, a_{i}\right)$ and $\left(\varsigma_{i^{\prime}}, a_{i^{\prime}}\right)$, then there are two sub-cases. In the first, $\left(\varsigma_{i^{\prime \prime}}, a_{i^{\prime \prime}}\right)$ is below $\left(\varsigma_{i}, a_{i}\right)$ and $\left(\varsigma_{i^{\prime}}, a_{i^{\prime}}\right)$ (i.e. $a_{i^{\prime \prime}} \leq a_{i^{\prime}}<a_{i}$ ). In this case $i^{\prime}$ could not be implementable as it would not be on the southwestern frontier. In the second sub-case $\left(\varsigma_{i^{\prime \prime}}, a_{i^{\prime \prime}}\right)$ is both horizontally and vertically between $\left(\varsigma_{i}, a_{i}\right)$ and $\left(\varsigma_{i^{\prime}}, a_{i^{\prime}}\right)$. Then $i^{\prime \prime}$ (or some other action also both horizontally and vertically between $\left(\varsigma_{i}, a_{i}\right)$ and $\left.\left(\varsigma_{i^{\prime}}, a_{i^{\prime}}\right)\right)$ would be implementable, contradicting that $i^{\prime}$ is the next lowest implementable action for $i$ 
Proof of Proposition 7: By Proposition 5, for any $k \geq 2, \varsigma_{k}-\varsigma_{k-1}<0$ and the minimal cost of implementing $k$ is $\varsigma_{k} c\left(e_{k}\right)=\varsigma_{k}\left(-\frac{a_{k-1}-a_{k}}{c_{k-1}-c_{k}}\right)$. A discrete version of the product and quotient rule for derivatives ${ }^{40}$ yields, for $k \geq 3$

$$
\begin{gathered}
C_{k}-C_{k-1}=\zeta_{k}\left(-\frac{\Delta a_{k}}{\Delta \zeta_{k}}\right)-\zeta_{k-1}\left(-\frac{\Delta a_{k-1}}{\Delta \zeta_{k-1}}\right) \\
=\Delta \zeta_{k}\left(-\frac{\Delta a_{k}}{\Delta \zeta_{k}}\right)+\zeta_{k-1}\left(-\frac{\Delta a_{k}}{\Delta \zeta_{k}}--\frac{\Delta a_{k-1}}{\Delta \zeta_{k-1}}\right) \\
=-\Delta a_{k}+-\zeta_{k-1}\left(\frac{\Delta a_{k}}{\Delta \zeta_{k}}-\frac{\Delta a_{k-1}}{\Delta \zeta_{k}}+\frac{\Delta a_{k-1}}{\Delta \zeta_{k}}-\frac{\Delta a_{k-1}}{\Delta \zeta_{k-1}}\right) \\
=-\Delta a_{k}+-\zeta_{k-1}\left(\frac{\Delta a_{k}-\Delta a_{k-1}}{\Delta \zeta_{k}}+\Delta a_{k-1}\left(\frac{1}{\Delta \zeta_{k}}-\frac{1}{\Delta \zeta_{k-1}}\right)\right) \\
=-\Delta a_{k}+-\zeta_{k-1} \frac{\Delta a_{k-1}}{\Delta \zeta_{k}}\left(\frac{\Delta a_{k}-\Delta a_{k-1}}{\Delta a_{k-1}}+\left(1-\frac{\Delta \zeta_{k}}{\Delta \zeta_{k-1}}\right)\right) \\
=-\Delta a_{k}+-\zeta_{k-1} \frac{\Delta a_{k-1}}{\Delta \zeta_{k}}\left(\frac{\Delta a_{k}-\Delta a_{k-1}}{\Delta a_{k-1}}+\frac{\Delta \zeta_{k}-\Delta \zeta_{k-1}}{-\Delta \zeta_{k-1}}\right) \\
=-\Delta a_{k}+\zeta_{k-1} \frac{\Delta a_{k-1}}{-\Delta \zeta_{k}}\left(\frac{\Delta^{2} a_{k}}{\Delta a_{k-1}}+\frac{\Delta^{2} \zeta_{k}}{-\Delta \zeta_{k-1}}\right) \cdot \square
\end{gathered}
$$

Proof of Proposition 8: First we provide some preliminary results on hearing payoffs. Given $\left(l_{1}, \ldots, l_{J} ; e_{1}, \ldots, e_{J}\right)$ define hearing payoffs $v_{j}=-l_{j}-j c\left(e_{j}\right)$. We say that evidence $e=\left(e_{1}, \ldots, e_{J}\right)$ implements $v=\left(v_{1}, \ldots, v_{J}\right)$ if it satisfies the hearing constraints with some $\left(l_{1}, \ldots, l_{J}\right)$ and for all $j, v_{j}=-l_{j}-j c\left(e_{j}\right)$. The following result is standard.

LEMMA 1: Evidence e implements $v$ if and only if $v_{j}-v_{j^{\prime}} \leq c_{j^{\prime}}\left(e_{j}\right)-c_{j}\left(e_{j}\right)$, all $j, j^{\prime}=1, \ldots, J$

Defining double differences $\Delta^{2} v=\left(\left(v_{1}-v_{2}\right)-\left(v_{2}-v_{3}\right), \ldots,\left(v_{J-2}-v_{J-1}\right)-\left(v_{J-1}-v_{J}\right),\left(v_{J-1}-v_{J}\right)\right)$, we have LEMMA 2: $v$ is implementable if and only if $\Delta^{2} v \geq 0$.

We show something more than LEMMA 2 and use the excess in proving LEMMA 4 to follow:

LEMMA 3: e implements $v$, if and only if $c\left(e_{1}\right) \geq v_{1}-v_{2} \geq c\left(e_{2}\right) \geq v_{2}-v_{3} \geq \ldots \geq c\left(e_{J}\right)$. 
Proof: Given LEMMA 1, the only issue is the sufficiency of checking adjacent types. Define $\forall j=1, \ldots, J, l_{j}=-v_{j}-j c\left(e_{j}\right)$. Then $\forall j, j^{\prime}=1, \ldots, J$ with $j<j^{\prime}, v_{j}-v_{j^{\prime}}$ $=\left(v_{j}-v_{j+1}\right)+\left(v_{j+1}-v_{j+2}\right)+\ldots+\left(v_{j^{\prime}-1}-v_{j^{\prime}}\right) \geq c\left(e_{j+1}\right)+\ldots+c\left(e_{j^{\prime}}\right) \geq\left(j^{\prime}-j\right) c\left(e_{j^{\prime}}\right)$. Substituting for $v_{j}$ and $v_{j^{\prime}}$ yields the result.

We may now prove Proposition 8 . With some manipulation we can rewrite the incentive constraints as $(G[i]-G) \Delta^{2} v \geq a[i]-a$, where $G$ is the $I \times J$ matrix whose $i^{\prime}$ th row is the vector of double cumulatives of $P_{i^{\prime}}, G[i]$ is the $I \times J$ matrix all of whose rows are identical and equal to the $i^{\text {th }}$ row of $G, a=\left(a_{1}, \ldots, a_{l}\right)$, and $a[i]=\left(a_{i}, \ldots, a_{i}\right) \in \mathfrak{R}_{+}^{l}$. Then $i$ is implementable if and only if a solution $\Delta^{2} v \in \mathfrak{R}^{J-1}$ exists to

$$
\left[\begin{array}{c}
G[i]-G \\
I
\end{array}\right] \Delta^{2} v \geq\left[\begin{array}{c}
a[i]-a \\
0
\end{array}\right]
$$

Applying Farkas' lemma, a solution exists if and only if there exist no $\pi \in \mathfrak{R}_{+}^{I}, q \in \mathfrak{R}_{+}^{J-1}$ such that $\pi^{\prime}(G[i]-G)+q^{\prime} I=0$ and $\pi^{\prime}(a[i]-a)>0$. The latter implies $\pi \neq 0$ and so we may take $\pi$ to be a probability measure. Rearranging, the existence of a solution to (17) is equivalent to the non existence of a probability measure $\pi$ such that $a_{i}>\pi^{\prime} a$ and $G_{i} \leq \pi^{\prime} G$

Proof of Proposition 9: Again, we start with some preliminary results. Let us say that $e$ implements $v$ at minimal cost, if it implements $v$ and for all other $e^{\prime}$ implementing $v$, $\sum_{j=1}^{J} P_{i j} c_{j}\left(e_{j}\right) \leq \sum_{j=1}^{J} P_{i j} c_{j}\left(e_{j}^{\prime}\right)$

LEMMA 4: If e implements $v$ at minimal cost, then $\forall j=1, \ldots, J-1$, $P_{i j}>0 \Rightarrow c\left(e_{j}\right)=v_{j}-v_{j+1}$ and $P_{i J}>0 \Rightarrow c\left(e_{J}\right)=0$. The minimum cost of implementing $v$ is 


$$
\sum_{j=1}^{J-1} P_{i j} j\left(v_{j}-v_{j+1}\right)=\sum_{j=1}^{J-1} \sum_{k=1}^{j} P_{i k} k \Delta^{2} v_{j}
$$

Proof: Suppose that for some $j \leq J-1, P_{i j}>0$ and $c\left(e_{j}\right)>v_{j}-v_{j+1}$. Since $\Re_{+} \subseteq c(E)$, we can find $e_{j}^{\prime} \in E$ such that $c\left(e_{j}^{\prime}\right)=v_{j}-v_{j+1}$ and then set $l_{j}^{\prime}=-v_{j}-j c\left(e_{j}^{\prime}\right)$. Then, by the lemma, $\left(l_{1}, \ldots, l_{j}^{\prime}, \ldots, l_{J}\right) \in \mathfrak{R}$ and $\left(e_{1}, \ldots, e_{j}^{\prime}, \ldots, e_{J}\right) \in \mathfrak{R}_{+}$implement $v$. But since $c\left(e_{j}^{\prime}\right)=v_{j}-v_{j+1}<c\left(e_{j}\right)$ and $P_{i j}>0,\left(l_{1}, \ldots, l_{j}^{\prime}, \ldots, l_{J}\right) \in \Re$ and $\left(e_{1}, \ldots, e_{j}^{\prime}, \ldots, e_{J}\right) \in \Re_{+}$are less costly than the hypothesized solution. Combining with the lemma yields $P_{i j}>0 \Rightarrow c\left(e_{j}\right)=v_{j}-v_{j+1}$. That $P_{i j}>0 \Rightarrow c\left(e_{J}\right)=0$ follows since, as shown in the lemma, implementation of $v$ imposes no lower bound on $c\left(e_{J}\right)$ and $c(E)=\mathfrak{R}_{+}$

Now consider the proposition. An (generically, the unique) optimum $\Delta^{2} v^{*}$ of the linear program: choose $\Delta^{2} v$ to minimize (18) subject to (17) will be at an extreme point of the feasible set. Therefore, the number of binding constraints at $\Delta^{2} v^{*}$ must be no less than $J-1$. There being $I$ constraints in the upper half of (17), the number of binding constraints in the lower half must be no less than $J-1-I$. This means that the number of non-binding constraints in the lower half must be no more than $(J-1)-((J-1)-I)=I$. Then any $v^{*}$ with second differences $\Delta^{2} v^{*}$ has no more than $I$ distinct first differences. By the lemma, in the minimum cost implementation of $v^{*}$, a positive probability type $j<J$ presents evidence whose cost equals $v_{j}^{*}-v_{j+1}^{*}$. Hence, among the positive probability types, there are no more than I distinct evidence costs. As is standard, evidence costs must be increasing in type and so types which present the same evidence must be adjacent. Lastly, it is clear that two types with equal evidence costs must incur equal liability. 


\section{REFERENCES}

Arlen J. (1992), "Liability for Physical Injury when Injurers as well as Victims Suffer Losses," Journal of Law, Economics and Organization, 8:411-426.

Bebchuk, L. (1984), "Litigation and Settlement Under Imperfect Information," RAND Journal of Economics, 15:404-415.

Brown J. (1973), “Toward an Economic Theory of Liability," Journal of Legal Studies, 2:323. Calabresi, G. (1972), The Costs of Accidents, New Haven, Yale University Press.

Daughety, A. and J. Reinganum (1994), "Settlement Negotiations with Two-Sided Asymmetric Information: Model Duality, Information Distribution, and Efficiency," International Review of Law and Economics, 14:283-298. and (1995), "Product Safety, Liability, R\&D, and Signaling," American Economic Review, 85:1187-1206.

Dewees, D., D. Duff, and M. Trebilcock, (1996) Exploring the Domain of Accident Law: Taking the Facts Seriously, New York, Oxford University Press.

Green, E. (1986), "Symposium: Probability and Inference in the Law of Evidence, Boston University Law Review, 66:377-952.

Froeb L. and B. Kobayashi (1993), "Competition in the Production of Costly Information: An Economic Analysis of Adversarial versus Court-Appointed Presentation of Expert Testimony," George Mason University, Law and Economics Working Paper \#93-005. (1995), "Naïve, Biased, yet Bayesian: Can Juiries Interpret Selectively Produced

Evidence," George Mason University, Law and Economics Working Paper \#95-008. 
Hermalin, B. and M. Katz (1991), "Moral Hazard and Verifiability: The Effects of Renegotiation in Agency," Econometrica, 59:1735-1753.

Holmstrom, B. and P. Milgrom (1991), "Multitask Principal-Agent Analyses" Journal of Law, Economics and Organization, 7:24-52.

Kaplow, L. and S. Shavell (1994) "Accuracy in the Determination of Liability," Journal of Law and Economics, 37:1-15. (1996), "Accuracy in the Assessment of Damages," Journal of Law and Economics, 39:191-210.

Kessler, D. and M. McClellan, (1996) "Do Doctors Practice Defensive Medicine," The Quarterly Journal of Economics, May 1996, 353-390.

Lipman, B. and D. Seppi, (1995) "Robust Inference in Communication Games with Partial Provability," Journal of Economic Theory, 66:370-405.

Milgrom, P. and J. Roberts (1986), "Relying on the Information of Interested Parties," RAND Journal of Economics, 17:1, 18-31.

Nalebuff (1987), "Credible Pretrial Negotiation," RAND Journal of Economics, 18:198-210.

Okuno-Fujiwara, M., A. Postlewaite and K. Suzumura (1990), "Strategic Information Revelation," Review of Economic Studies, 57:25-47.

Ordover, J. (1978), "Costly Litigation in the Model of Single Activity Accidents," Journal of Legal Studies, 7:243-261. (1981), "On the Consequences of Costly Litigation in the Model of Single Activity Accidents: Some New Results," Journal of Legal Studies, 10:269-291.

Png, I. (1983), "Strategic Behavior in Suit, Settlement and Trial," Bell Journal of Economics 14:539-550. 
(1987), "Litigation, Liability, and Incentives for Care," Journal of Public Economics

$34: 61-85$.

Polinsky, A. and Y. Che (1991), "Decoupling Liability," RAND Journal of Economics, 562. . and D. Rubinfeld (1988), "The Welfare Implications of Costly Litigation for the Level of Liability," Journal of Legal Studies, 17:151-164.

Rubinfeld, D. and D. Sappington (1987), "Efficient Awards and Standards of Proof in Judicial Proceedings," RAND Journal of Economics, 18:2, 308-315.

Sanchirico, C., (1996), "Enforcement by Hearing: How the Civil Law Sets Incentives,"

Columbia Economics Department, Discussion Paper No. 95-9603. (1997), "Evidence Production, Adversarial Process and the Private Instigation of Suits," mimeo Columbia Economics Department, available at www.columbia.edu/ $\sim \operatorname{cs} 282$

Shavell, S. (1980), "Strict Liability versus Negligence," Journal of Legal Studies, 9:1. (1989), "Sharing of Information Prior to Settlement or Litigation," RAND Journal of Economics 20:183-195. (1996), "The Level of Litigation: Private versus Social Optimality," Harvard Law School Working Paper

Sobel, J. (1985), "Disclosure of Evidence and Resolution of Disputes," in A. E. Roth, ed. Game Theoretic Models of Bargaining, 341.

Spence, M. (1974), Market Signaling, Cambridge, MA, Harvard University Press.

Spier, K. (1994a), "Pretrial Bargaining and the Design of Fee Shifting Rules," RAND Journal of Economics, 25:197-214. (1994b), "Settlement Bargaining and the Design of Damage Awards," Journal of Law Economics and Organization, 10:84-95. 
Townsend (1979), "Optimal Contracts and Competitive Markets with Costly State Verification," The Journal of Economic Theory, 21:265-293 
${ }^{1}$ I thank seminar participants at the 1997 Stanford Institute for Theoretical Economics (SITE), the 1995 and 1997 meetings of the American Law and Economics Association, the Columbia University Economics Department and Law School, George Mason Law School, Stanford Law School, the University of Southern California Law Center, and the California Institute of Technology. I have had helpful conversations with Ron Miller, Andrew Newman, Alexander Pfaff, Mitchell Polinsky, Michael Riordan, Kathy Spier, and Eric Talley. I acknowledge financial support from Yale Law School's Career Options Assistance Program and the Olinsponsored Law and Economics Programs at U. C. Berkeley's Jurisprudence and Social Policy Program, Stanford Law School and Columbia Law School.

${ }^{2}$ This draft is based on the first half of the December 1995 draft (listed in the references as Sanchirico (1996)). Ideas presented in the second half of the December 1995 draft concerning multiple parties and privately instigated suits appear in a separate paper, Sanchirico (1997), which is available at www.columbia.edu/ $\operatorname{cs} 282$.

${ }^{3}$ Litigation and tort reform was, for example, an important component of the Republican Congress" "Contract with America." For a general and timely review of the debate, see Dewees, Duff and Trebilcock (1997).

${ }^{4}$ This is not justified by the fact that most cases settle, since settlement occurs in the shadow of parties expectations about what would happen were the case to go to trial.

${ }^{5}$ See, e.g., Calabresi (1972), Brown (1973), Shavell (1980), Ordover (1978,1981), Polinsky and Rubinfeld (1988), Arlen (1992), Spier (1994b), Shavell (1996).

${ }^{6}$ See, e.g., Png (1983, 1987), Bebchuk (1984), Nalebuff (1987), Shavell (1989), Daughety and Reinganum (1994), Spier (1994a), Shavell (1996). 
${ }^{7}$ See, e.g., Kaplow and Shavell (1994 and 1996).

${ }^{8}$ See Rubinfeld and Sappington (1988).

${ }^{9}$ I thank Michael Riordan for suggesting the term "reduced form" in this context.

10 "Civil" process is that by which private parties sue each other. The "opposite" here is criminal process whereby the state brings actions against private parties.

${ }^{11}$ See the literature following Townsend (1979). That reduced form trials are variants of costly state verification models is perhaps clearest in papers such as Polinsky and Rubinfeld (1988), Polinsky and Che (1991) and Kaplow and Shavell (1994).

${ }^{12}$ For a recent review of this debate in Canada and the U.S., see Dewees, Duff and Trebilcock (1996). All three authors are at the University of Toronto.

${ }^{13}$ The overdeterrence critique is most prevalent in the areas of medical malpractice and products liability. See Dewees, Duff and Trebilcock (1996), p. 6 and references therein. ${ }^{14}$ That this is how Kessler and McClellan's (1996), for instance, define "defensive medicine," might be gleaned from what is left out of the following statement:

"Identifying the existence of defensive treatment practices and the extent of inefficient precaution due to legal liability requires a comparison of the response of costs of precaution and the response of losses from adverse events to changes in the legal environment" ( $p$. 360).

${ }^{15}$ It is of course incorrect to rejoin that since implementation costs are dwarfed by the costs of precaution and accidents, they can be effectively neglected. Regardless of whether the empirical claim is true, margins, not levels determine both the relative size of first and second best as well as the losses from employing the first best level in a world with varying implementation costs. Thus, translating the U-shaped curve in Figure 1 upward has no effect on the difference between first and second best, nor on the losses incurred by ignoring implementation costs. Of course, 
the claim that implementation costs are small in an absolute sense would bear on the issue, but if there is any consensus on the size of these costs, it is for the opposite assertion. ${ }^{16}$ Specifically, we apply Spence's idea of differential cost signaling (as opposed to cheap talk signaling). There is no analogy here to the market structure in Spence's model.

${ }^{17}$ For an example of a model in another context in which hidden actions determine type in a subsequent signaling game, see Daughety and Reinganum (1995). In that model R\&D expenditure is the hidden action, product safety is the type and, depending on the products liability regime in place, price may act as a signal of safety to consumers.

As suggested to us by Andrew Newman, such models can also be thought of as multi-task principal agent problems with varying observability across tasks. Holmstrom and Milgrom (1991), however, emphasize the danger of substitution into the observed and rewarded action at the expense of the unobserved action. Recall their example in which rewarding school teachers for student test scores causes teachers to shift effort into training for test taking and out of training for creativity. In contrast, the model we propose in this paper operates via complementarities as between the observable action (evidence production) and the unobservable action (care level).

${ }^{18}$ I thank Kathy Spier for suggesting this reinterpretation. See also Corollary 1 in Sanchirico (1996).

${ }^{19}$ Throughout, I denote a finite set and the number of its elements with the same symbol. ${ }^{20}$ Even though $p$ is fixed across actions, our specification allows for the case in which each action produces a different distribution over evidence cost schedules. Let $J$ index the set of all possible evidence cost schedules. Set $S$ to equal the set of all $I$-vectors $\left(j_{1}, \ldots, j_{I}\right)$, each $j_{i} \in J$. Let $c_{i s}(e)=c_{j_{i}}(e)$. The measure $p$ is then the joint distribution across action choices of 
evidence costs. Any collection of possibly different marginal distributions, one for each action $i$, can be created in this manner (see Section 5 , for example). What's important in this formulation is that the agent observes $s$ separately from $i$, which implies that the agent can imagine what would have happened under the same circumstances had she taken a different action.

${ }^{21}$ A liability schedule $l(e)$ corresponding to this direct mechanism: no liability if evidence of at least 1 is presented; otherwise liability of -100 . For the general correspondence between vectors $\left(l_{1}, \ldots, l_{l}, e_{1}, \ldots, e_{l}\right)$ of liability and evidence assignments and liability per evidence functions $l(e)$, see Proposition 10.

${ }^{22}$ Indeed in the deterministic case, for general evidence cost functions, $i$ is implementable if and only if it is implementable with all care levels presenting the same evidence (Corollary 2). This is not generally true for the stochastic case; examples are easy to construct.

${ }^{23}$ A remark similar to note 22 applies here. That such simple schedules are not generally optimal in the stochastic case is shown in Proposition 9.

${ }^{24}$ To fit this into a litigation framework, we might imagine, as in Polinsky and Che (1991), that harm to victims is a random variable observable by the judge in court. Then the amount we award as a function of harm determines which plaintiffs are willing to pay their litigation costs and sue and thus also the likelihood that we "audit" the injurer.

${ }^{25}$ The comparison of the random audit mechanism with our own raises the question of what would happen if we randomly held hearings in our model. The somewhat counterintuitive answer is that nothing happens: the minimal cost of implementation is still $C_{i}$ from (11) and so the second best optimum is still maximal care $i=10$. The reason nothing happens is that there is in fact no cost advantage to reducing the audit probability in our model. For to the extent we 
hold hearings less often, each hearing that we do hold must be that much more expensive. This is of course a direct consequence of the fact that we are being explicit about how these hearings work, instead of simply assuming that information is revealed whenever such a hearing occurs. More specifically, if we add randomly held hearings to our model, (6) becomes $q\left(v_{i}-v_{i^{\prime}}\right) \geq a_{i}-a_{i^{\prime}}$ where $q \in[0,1]$ is now a choice variable for the court. Of course, we must set $q>0$ to implement any $i \geq 2$. An analysis similar to that conducted in subsections 2.1 and 2.2 above shows that, with any fixed $q>0$, the minimal cost evidence liability per evidence schedule sets $e_{i}=\frac{1}{q}$ for any $i \geq 2$. Thus the minimal cost of implementing action $i \geq 2$ with fixed $q$ is $q c_{i}\left(e_{i}\right)=q(11-i) \frac{1}{q}=11-i$, just as in (11).

The minimal cost of implementation schedule is merely translated upwards (over $i \geq 2$ ) if we add a fixed cost $k>0$ for hearings (whether or not $k$ is born in the first instance by the agent). For given $q$, optimal evidence will still entail $e_{i}=\frac{1}{q}$ and so expected evidence costs will still be constant in $q$ at $(11-i)$. However, total expected costs will now be $q k+(11-i)$. It will then be optimal to reduce $q$ as much as possible and for a solution to exist we will need to bound all $v_{i^{\prime}}$ and $l_{i^{\prime}}$ from below, again positing the existence of solvency constraints. Importantly, such constraints will not change depending on which action we happen to be implementing. Thus, the lowest attainable and hence optimal $q$, call it $q$, will be the same no matter what action we are implementing. The minimal cost of implementation will then be $q k+(11-i)$, a translation of the solid line in Figure 3 over $i \geq 2$. Hence, in the set $i \geq 2, i=10$ will still be optimal. Whether $i=10$ still beats $i=0$ will depend on the size of $q k$. ${ }^{26}$ These approaches are identified in the introduction. 
${ }^{27}$ Note that evidence $e$ has this property for target care level $i$ if and only if $c_{i}(e)$ is finite and $c_{i^{\prime}}(e)$ is infinite for all $i^{\prime} \neq i$. This justifies our definition of perfect evidence. In contrast, evidence which is costless for $i$ and costly for all other $i^{\prime}$ does not have this property. ${ }^{28}$ With respect to the coarsest topology on $E$ under which the vector valued function $\left(c_{11}, \ldots, c_{I S}\right)$ is continuous.

${ }^{29}$ If action $i$ is not implementable then $C_{i}$ is infinite. We will be describing the slope as between implementable actions.

${ }^{30}$ Note that I-1 may not be implementable.

${ }^{31}$ We simply add and subtract the term $\sum_{s=1}^{S} p_{s} c_{i^{\prime} s}\left(e_{i s}\right)$.

${ }^{32}$ This is an additional assumption that is not part of the usual meaning of multiplicative separability.

${ }^{33}$ Here we use the term "convexity" to mean a "non negative second difference." Thus $x_{1}, \ldots, x_{l}$ is convex if for all $i=3, \ldots, I,\left(x_{i}-x_{i-1}\right)-\left(x_{i-1}-x_{i-2}\right)=\Delta x_{i}-\Delta x_{i-1}=\Delta^{2} x_{i} \geq 0$. The adjective "relative" means that we take percentage changes.

${ }^{34}$ Note that $c_{i}(e)$ is strictly decreasing and convex in $i$ for each fixed $e \in E$ if and only if $\varsigma_{i}$ is strictly decreasing and convex in $i$.

${ }^{35}$ Recall that actions are ordered according to their private costs.

${ }^{36}$ Neither is necessary for convexity of private costs in evidence costs. For instance, private costs may accelerate fast enough to outweigh an accelerating decline in evidence costs.

${ }^{37}$ As in the example in Section 2 (see note 25), allowing for randomized hearings does not affect the results in this section: all propositions continue to hold as written. Arguments similar to those in this section's proofs establish that for any fixed hearing probability $q$, action $i$ presents 
evidence $c\left(e_{i}\right)=-\frac{1}{q} \frac{a_{r}-a_{i}}{s_{r}-s_{i}}$ in a minimal cost implementation of $i$. Thus the minimum cost of implementing action $i$ given $q$ is $q c\left(e_{i}\right)=q\left(-\frac{1}{q} \frac{a_{i}-a_{i}}{s_{i}-\zeta_{i}}\right)=-\frac{a_{i}-a_{i}}{\zeta_{i}-\zeta_{i}}$. Since, this is not a function of $q$, randomization is of no consequence.

${ }^{38}$ This is implicit in Corollary 2. That result says that action $i$ is implementable if and only if it is implementable with all actions presenting the same evidence. Since only the implemented action's costs matter, the minimal cost liability may as well be uniform.

${ }^{39}$ That the $\varsigma_{\text {is }}$ are positive integers is without loss of generality. If all $\varsigma_{\text {is }}$ are rational numbers, redefine $c(e)$ by dividing it by the least common denominator of the $\varsigma_{i s}$, each represented as an integer fraction. If a given $\varsigma_{\text {is }}$ is not rational, we can approximate it with a rational number. All the results that we present here can then be proven by passing to the limit of the approximating sequence of rationals.

${ }^{40}$ The reader may at this juncture wonder why we have not employed a model with a continuum of actions. This would make taking this particular "derivative" much easier, but it would necessitate the first order approach in solving the mechanism design problem which in turn requires assumptions on second and third cross partials that are difficult to justify. 


\title{
1997-1998 Discussion Paper Series
}

\author{
Department of Economics \\ Columbia University \\ 1022 International Affairs Bldg. \\ 420 West 118th Street \\ New York, N.Y., 10027
}

The following papers are published in the 1997-98 Columbia University Discussion Paper series which runs from early November to October 31 of the following year (Academic Year).

The following is a website address where viewers may download some discussion papers. It is a project that is in progress, and therefore the site may not contain all recent working papers.

http://www.columbia.edu/dlc/wp/econ/index.html.

For Ordering Hardcopies:

Domestic orders for discussion papers are available for purchase at the cost of $\$ 8.00$ (U.S.) Per paper and $\$ 140.00$ (US) for the series.

Foreign orders cost $\$ 10.00$ (US) per paper and $\$ 185.00$ for the series.

To order discussion papers, please write to the Discussion Paper Coordinator at the above address along with a check for the appropriate amount, made payable to Department of Economics, Columbia University. Please be sure to include the series number of the requested paper when you place an order. 
1997-98 Discussion Papers

Economics Department, Columbia University

\begin{tabular}{|l|l|l|}
\hline Series no: & \multicolumn{1}{|c|}{ Tite } & \multicolumn{1}{|c|}{ Authors } \\
\hline $9798-01$ & Optimal Regulatory Transparency & Herk, L.F. \\
\hline $9798-02$ & Russia's Market Transition: Who Has Lost and How? & $\begin{array}{l}\text { Desai, P. } \\
\text { Idson, T. }\end{array}$ \\
\hline $9798-03$ & The Wage Arrears Crisis in Russia & $\begin{array}{l}\text { Desai, P. } \\
\text { Idson, T. }\end{array}$ \\
\hline $9798-04$ & Taxes Versus Legal Rules as Instruments for Equity & Sanchirico, C.W. \\
\hline $9798-05$ & Enforcement by Hearing: An Integrated Model of Evidence Production (first draft: 12/95) & Sanchirico, C.W. \\
\hline $9798-06$ & $\begin{array}{l}\text { Evidence Production, Adversarial Process and the Private Instigation of Suits (first draft: } \\
12 / 95)\end{array}$ & Sanchirico, C.W. \\
\hline
\end{tabular}

